\title{
Metaberichterstattung. Medienselbstthematisierung und Publicity-Thematisierung in amerikanischen, britischen und deutschen Wahlkämpfen
}

Esser, Frank

Other titles: Metacoverage. Media self-coverage and publicity coverage in American, British and German election campaigns

Posted at the Zurich Open Repository and Archive, University of Zurich

ZORA URL: https://doi.org/10.5167/uzh-8074

Book Section

Originally published at:

Esser, Frank (2008). Metaberichterstattung. Medienselbstthematisierung und Publicity-Thematisierung in amerikanischen, britischen und deutschen Wahlkämpfen. In: Melischek, G; Seethaler, J; Wilke, J. Medien Kommunikationsforschung im Vergleich. Grundlagen, Gegenstandsbereiche, Verfahrensweisen. Wiesbaden: VS Verlag, 121-156. 


\title{
Metaberichterstattung: Medienselbstthematisierung und Publicity-Thematisierung in amerikanischen, britischen und deutschen Wahlkämpfen
}

\author{
Frank Esser
}

\section{Grundlagen der Mediatisierung}

Der Prozess der Mediatisierung verweist auf die zunehmende Bedeutungssteigerung der Massenmedien für die politische Kommunikation. ${ }^{1}$ Diese Entwicklung, in der "the media [are] moving toward the center of the social process" (Blumler/Kavanagh 1999: 211), begünstigt den Modern Publicity Process, worunter Jay Blumler eine wachsende Intervention der Medien in politische Vorgänge versteht, was zwischen Journalisten und Politikern einen „competitive struggle to influence and control popular perceptions of key political events and issues through the mass media" auslöst (Blumler 1990: 103). Der Beziehungsstress entzündet sich am Bedürfnis beider Akteursgruppen, die Kommunikationskontrolle über die öffentlich zirkulierenden Botschaften und Themen - gerade im Wahlkampf - zu behalten.

Im Wettbewerb um das knappe Gut Aufmerksamkeit müssen sich Politiker an der Selektions- und Präsentationslogik der Massenmedien orientieren, um öffentlichkeitswirksam zu bleiben. Sie versuchen dabei einerseits, die Medienlogik für ihre Zwecke zu instrumentalisieren, und sehen andererseits die Notwendigkeit, sich den Bedürfnissen der Medien anzupassen. Daher versuchen sie, mittels politischer Öffentlichkeitsarbeit geeignete Ereignisse und Themen bereits auf die Medienlogik hin zu trimmen. Die daraus resultierende Zunahme an mediatisierten Ereignissen und inszenierten Themen hat weitreichende Konsequenzen: Das Politikbild, das die Medien heute darstellen, ist in erheblichem Maße eine zum Zwecke der Berichterstattung kreierte Realität - Medienwirklichkeit und soziale Realität verschmelzen (Kepplinger 1998: 173; Sarcinelli 1998: 678f.). Das Geschehen und seine Darstellung formen ein rückgekoppeltes System, dessen Selbstreferenzialität - also dessen Berichterstattung über bereits mediatisierte Ereignisse - zunimmt. Dieser Prozess, den Altheide und Snow in "Media Worlds in the Postjournalism Era“ (1991) erstmals ausführlich untersuchten, wird von den Publicity-Strategen der Politiker zunehmend einkalkuliert und führt im Bereich medienzentrierter

\footnotetext{
${ }^{1}$ Dem auch verbreiteten Begriff Medialisierung wird hier der international eingebürgerte Terminus Mediatisierung (vgl. Mazzoleni/Schulz 1999) konsequent vorgezogen, ohne dass damit ein inhaltliches Statement verbunden wäre.
} 
Kampagnenkommunikation zu „postmodernen Wahlkämpfen“, auf die Journalisten u.a. mit reflexiver „Metaberichterstattung“ reagieren (Plasser/Plasser 2003: 17, 106).

Theoretisch gesprochen wird politische Kommunikation vor diesem Hintergrund als Interpenetrationsprodukt von Politik und Medien begriffen (Jarren/Donges 2006). Politische Themen sind das Ergebnis der Interaktions- und Austauschprozesse zwischen politischen und journalistischen Akteuren. Diese Austauschprozesse institutionalisieren sich mit zunehmender Dauer $\mathrm{zu}$ einem (empirisch beobachtbaren) Handlungssystem, bei dem sich formalisierte Formen der Interaktionen (z.B. Pressekonferenzen) und informelle Formen (z.B. Hintergrundgespräche und Gesprächszirkel) unterscheiden lassen. In diesem Handlungssystem der politischen Kommunikation durchdringen sich Politik- und Medienlogik wechselseitig. Der Prozess der Mediatisierung beschreibt nun, inwieweit es in diesem Handlungssystem (bestehend aus den Akteuren der politischen Publicity und des politischen Journalismus) „strukturell“ zu Zuwächsen von Medien- und Publicity-Ressourcen kommt (z.B. durch neue zentrale Akteurstypen, Tätigkeitsbereiche, Institutionengefüge) und inwieweit es „kulturell“ von der Medienlogik im Verhältnis zur Politiklogik bestimmt ist (z.B. wie sehr die Selektions- und Präsentationsregeln der Medien als Geschäftsgrundlage der Interaktionsbeziehungen anerkannt sind). Damit verweist der Mediatisierungsprozess auf den Struktur- und Kulturwandel in politischen Kommunikationssystemen verschiedener Länder; diese politischen Kommunikationssysteme sind durch einen organisatorischinstitutionellen (strukturell) sowie einstellungs- und verhaltensrelevanten (kulturell) Bedeutungsgewinn von Medien- und Publicity-Aspekten gekennzeichnet ist. ${ }^{2}$

Mit steigendem Mediatisierungsgrad nimmt auch die Selbstbezüglichkeit des politischen Kommunikationssystems zu. Dies zeigt sich beispielsweise in der wachsenden Neigung von PR- und Publicity-Beratern, nicht mehr die Maximen des Politiksystems (das sie rekrutiert) zu ihrer wesentlichen Handlungsorientierung zu machen, sondern sich an der stark mediatisierten Mischlogik des politischen Kommunikationssystems zu orientieren. Dies führt zu der postmodernen Entwicklung, dass sich früher verdeckt arbeitende Kandidatenberater plötzlich auf die Vorderbühne drängen, um sich vor TV-Kameras zum Inszenieren und Kulissenschieben zu bekennen. Bergmann (2002: 142) kommt in seiner Analyse des SPDWahlkampfes 1998 zu dem Schluss, dass ein zentraler Baustein der Kampa-Kommunikationsstrategie darin lag, Medienberichte über die Kampa selbst zu initiieren. Laut Wahlkampfleiter Malte Ristau tut man „der Kampa wohl kein Unrecht,

2 Dieses Verständnis von Mediatisierung ist stark beeinflusst von Blumler/Gurevitch (1995), Donges (2005), Jarren/Donges (2006), Pfetsch (2003). 
wenn man festhält, dass die Metakommunikation wichtiger war als der reale Output“. Mit diesem Wandel der Kampagnenführung ging auch ein spürbarer Wandel der Kampagnenberichterstattung einher (Fengler/Jun 2003: 188; Röseler 2003: 201f.). Er führte im deutschen Bundestagswahlkampf 2002 u.a. zu einer Fülle von Insider-Reportagen über die Wahlkampfzentralen, die Fernsehteams einluden, sie bei der Kreierung eines Medienwahlkampfes zu filmen. ${ }^{3}$ Sarcinelli (2000: 29) fordert zur Analyse dieses „bisher vernachlässigten Aspekts“ auf, „der Metakommunikation, also der Kommunikation über die Kommunikation. Denn es scheint so, dass der Wettbewerb um das medien- und wählerwirksamste Politikmarketing, der Wahlkampfstil, die Politikvermittlung selbst zum Thema der Politikvermittlung wird." Zur Tendenz der Medien, die Grundlagen der Berichterstattung zum Gegenstand der Berichterstattung machen, stellten Moog und Slyter-Beltro (2001: 38) fest, dass "political communication has become increasingly hyper-reflexive“. Denselben Trend haben McNair (2000: 171) für Großbritannien als „meta-discursive phase“ und Neveu (2002: 31) für Frankreich als „kind of meta journalism“ beschrieben.

\section{Grundlagen der Metaberichterstattung}

Mediatisierung ist kein Hinterbühnenvorgang, sondern vollzieht sich öffentlich und öffentlichkeitswirksam - auf der Vorderbühne. So zeigen Medieninhaltsanalysen US-amerikanischer Präsidentschaftswahlkämpfe, dass die Konturen mediatisierter Politik zunehmend zum Nachrichtenthema werden, indem beispielsweise über die Medienbeziehungen der Kandidaten, ihre Versuche der Informationssteuerung oder ihre Strategien der Auftrittsinszenierung und Wahlwerbung berichtet wird. Dieser Berichterstattungstyp wird als self-referential process news (Kerbel 1998), media process news (Kerbel et al. 2000), coverage of coverage (Gitlin 1991) oder stories about the media (Johnson et al. 1996) bezeichnet. Nach einer längeren Auseinandersetzung mit der Literatur erscheint mir der Ausdruck „Metaberichterstattung" am angemessensten. Darunter verstehe ich Berichterstattung über Wahlkämpfe, bei der die Rolle der Medien selbst oder die Rolle von politischer Publicity hervorgehoben wird. Damit umfasst Metaberichterstattung zwei Dimensionen: Medienselbstthematisierungen und Publicity-Thematisierungen. ${ }^{4}$

\footnotetext{
${ }^{3}$ Vgl. u.a. „Die Wahlkampfmacher“ (ARD vom 28.8.2002, 23:30 Uhr), „Die Kanzlermacher“ (RTL vom 5.9.2002, 0:15 Uhr), „Die Kanzlermacher“ (n-tv vom 15.9.2002, 20:15 Uhr), "Das große Schauspiel“ (Phoenix vom 16.9.2002, 20:15 Uhr), „Die Kanzlermacher“ (ZDF vom 11.11.2002, 23:50 Uhr).

${ }^{4}$ "Selfreferential metacoverage" und "process metacoverage“ (Esser et al. 2001) bzw. "press metacoverage" und "publicity metacoverage“ (Esser/D’Angelo 2003).
} 
Medienselbstthematisierungen umfassen ein breites Spektrum möglicher Erwähnungen von Akteuren, Handlungsweisen, Normen oder Produkten des Journalismus. Wenn zum Beispiel anwesende Reporter, der Einfluss von Bild-Chefredakteur Kai Diekmann, die Duell-Vorbereitungen von ARD-Moderatorin Christiansen, Diskussionen in der Redaktion der Financial Times Deutschland über eine Wahlempfehlung, Klagen über eine vermeintlich einseitige Spiegel-Berichterstattung oder eine Verteidigung des Ausgewogenheitsprinzip beim ZDF in einem Wahlkampfbeitrag diskutiert werden, liegen Medienselbstthematisierungen vor. Publicity-Thematisierungen umfassen u.a. Erwähnungen von Akteuren, Handlungsweisen, Zielen oder Strategien der politischen Werbung oder Öffentlichkeitsarbeit. Wenn in einem Wahlkampfbeitrag z.B. Auftrittsplanung, Choreographie, Kameratauglichkeit, Kommunikationsdisziplin oder Selbstdarstellerqualitäten eines Politikers, die Arbeit seiner Kommunikationsberater und PR-Strategen, Tätigkeiten wie Themen-, Image-, Ereignis- und Newsmanagement oder Werbe- und Marketingmaßnahmen behandelt werden, liegen Publicity-Thematisierungen vor.

Hinsichtlich der normativen Bewertungen von Metaberichterstattung werden unterschiedliche Einschätzungen vertreten. Eine in den USA sehr prominente Position stammt von Kerbel (1997: 97ff.; 1999: 83ff.). Er sieht Metaberichterstattung als problematisch an, weil sie keinen Beitrag zur informierten Wahlentscheidung, zum demokratischen Problemdiskurs und zum öffentlichen Meinungsbildungsprozess leistet. Im Gegenteil, das Hochspielen von Inszenierungsbemühungen, von Machtspielchen zwischen Journalisten und Politikern sowie von Selbstumkreisungen der Journalistenseele entziehe der sehr viel notwendigeren Berichterstattung über substanzielle Inhalten wertvollen Raum. Zudem schüre die Präsentation des Kampagnenprozesses als theatraler Schaukampf von posierenden Rollenspielern in der Öffentlichkeit nur Zynismus und Politikverdrossenheit. Eine völlig entgegengesetzte Position vertritt McNair (2000: 46, ff., 122, ff., 171, ff.). Er begrüßt Metaberichterstattung als Entschlüsselungsjournalismus, der einen wesentlichen Beitrag zur Transparenz und Aufklärung in der demokratischen Öffentlichkeit leistet. Mit der Berichterstattung über die veränderten Bedingungen politischer Kommunikation würde Metaberichterstattung den Wählern einen wertvollen Dienst erweisen. Indem Journalisten die Marketinglogik und Inszenierungsbühne offen legten, könnten Bürger informiertere Entscheidungen über moderne Politik treffen. Eine dritte, sehr neutrale Einschätzung haben Johnson et al. (1996) vorgetragen. Für sie dokumentiert Metaberichterstattung lediglich die faktische Bedeutungssteigerung, die dem Nachrichtenjournalismus als zentraler Vermittlungsinstitution im Wahlkampf zukommt. Sie signalisiert weitgehend wertfrei, welche Kommunikationskanäle die Kandidaten für die Verbreitung ihrer Botschaften verwenden. 
Der vorliegende Beitrag vertritt die Auffassung, dass Metaberichterstattung in allen drei Ausformungen vorkommen kann. Dabei knüpft er an Befunde der Pilotstudie von Esser und D'Angelo (2003; D'Angelo/Esser 2003) an, die zeigen, dass in den amerikanischen Fernsehnachrichten drei Medien-Frames und drei PublicityFrames vorherrschen, mit denen Fernsehjournalisten die Rolle der Nachrichtenmedien und der Publicitymaßnahmen im Wahlkampf thematisieren. Wie dies konkret diskursiv geschieht, konnten sie mit einer Framinganalyse erklären. Das Ziel der vorliegenden Untersuchung liegt darin, die Studie von Esser und D'Angelo (2003; D'Angelo/Esser 2003) auszuweiten, indem die amerikanischen Befunde hier nun mit britischen und deutschen verglichen werden. Die Grundannahme lautet, dass der strukturelle und kulturelle Mediatisierungsgrad eines nationalen politischen Kommunikationssystems die Intensität und Frameverteilung der Metaberichterstattung in jeweiligen nationalen Leitmedien beeinflusst.

Zur Bestimmung des kulturellen Mediatisierungsgrades greifen wir auf eine ländervergleichende Befragung politischer Journalisten und politischer PublicityExperten von Pfetsch (1998; 2003a; 2003b) zurück. Sie konnte zeigen, dass deren Interaktionen in den USA durch eine deutlich medienorientierte Kommunikationskultur bestimmt wird, während in Großbritannien und Deutschland noch eine eher parteipolitisch geprägte Kommunikationskultur vorherrscht. Dafür macht sie vor allem strukturelle Charakteristika verantwortlich. So sei in den USA die Stellung der Parteien schwächer, dafür die des politischen Kommunikationsmanagements stärker. In Großbritannien und Deutschland ist die politische Kommunikation dagegen stärker von ideologischen Schemata bestimmt, wofür die starken Parteiinstitutionen und die ausgeprägteren politischen Linien in der meinungsführenden Presse typische Anzeichen sind. ${ }^{5}$ Noch deutlicher wird der unterschiedliche Mediatisierungsgrad im Wahlkampf. Hier gelten die USA aufgrund der zentralen

\footnotetext{
${ }^{5}$ Nach Pfetsch (2003) stehen in Deutschland (und vermutlich analog in Großbritannien) erstens weiterhin weltanschaulich verwurzelte Parteien im Zentrum des politischen Prozesses, zweitens säße Parteipersonal an den Schlüsselpositionen der politischen Publicity und Wahlkampfführung, drittens positionierten sich meinungsführende Zeitungen entlang des politischen Rechts-Links-Spektrums, viertens seien die einflussreichen Hintergrundkreise und informellen Netzwerke zwischen Politikern und Journalisten durch ideologische Nähe geprägt, und fünftens seien die öffentlich-rechtlichen Rundfunkanstalten in Programm- und Personalpolitik parteiisch orientiert. Während die Interaktionsbeziehungen zwischen Politik und Presse nach Pfetschs Befunden in Deutschland durch eine stärker parteipolitischideologisch motivierte Kommunikationskultur geprägt sind, bestimmt in den USA die Medienlogik die Geschäftsgrundlage der kulturellen Beziehungen. Der Mediatisierungsgrad des politischen Kommunikationssystems sei in den USA aus folgenden strukturellen Gründen höher: Durch die schwächere Stellung der Parteien seien für politische Akteure die Medien die entscheidende strategische Ressource für effektive politische Handlungsfähigkeit. Weil sich öffentliche Unterstützung und Issue-bezogene Mobilisierung nur mittels medienvermittelter Kommunikation erreichen ließe, seien Politiker in besonderer Weise auf Journalisten angewiesen.
} 
Stellung ihres durchkommerzialisierten Rundfunksystems, der unbeschränkten Möglichkeit zum Kauf politischer TV-Werbezeiten und der Dominanz politischer Marketingstrategien "als Prototyp einer medienzentrierten Demokratie“ (Filzmaier/Plasser 2001: 137). ${ }^{6}$ Die amerikanischen Medien haben sich zu einem politischen Schlüsselakteur für Regierungs- und Wahlkampfführung entwickelt (Patterson 1993; Graber 2007).

Mit diesen Überlegungen folgen wir den von Gurevitch und Blumler (2003: 380f.) ausformulierten Qualitätskriterien für ausgereifte Vergleichsstudien: Wir haben erstens eine Zielsetzung formuliert (Generalisierung und Kontextualisierung des am US-Material entwickelten Metaberichterstattungskonzepts); wir haben zweitens einen theoretischen Rahmen entworfen (politisches Kommunikationssystem); wir haben drittens eine zentrale Grundannahme postuliert (dass die strukturellen und kulturellen Bedingungen des politisches Kommunikationssystems einen Einfluss auf Metaberichterstattung haben); wir werden auf dieser Basis konkrete Hypothesen entwickeln und die Länderauswahl begründen; und wir werden schließlich fünftens auf Basis unserer Ergebnisse Schlussfolgerungen für die Gültigkeit des Metaberichterstattungskonzepts ziehen (was gilt universell, was ist kontextabhängig).

\section{Metaberichterstattung als Thema und Frame}

Eine Diskussion über Metaberichterstattung aus Perspektive der politischen Kommunikationsforschung muss bei den Vorarbeiten von Matthew Kerbel (1997; 1998; 1999; Kerbel et al. 2000) ansetzen. Er argumentiert, dass Medien- und PublicityThematisierungen in der Wahlkampfberichterstattung erstens zu einer Verdrängung substanzieller Policy-Themen führen; und dass Medien- und PublicityThematisierungen zweitens bevorzugt in einem Strategie- bzw. Game-Kontext von Journalisten verwendet werden (v.a. weil die Strategie-Orientierung die Wahl-

\footnotetext{
${ }^{6}$ Nach Plasser/Plasser (2003) führte der Bedeutungsverlust amerikanischer Parteien zu einer Verdrängung parteienzentrierter Wahlkampfstile durch fernseh- und kandidatenzentrierte Kampagnentechniken. $\mathrm{Zu}$ diesen gehören unbegrenzt einkaufbare TV-Werbezeiten sowie das Fernsehen als zentrale Plattform für Kampagnenauftritte (z.B. in TV-Debatten oder Unterhaltungssendungen). Das präsidentielle Regierungssystem führt zu von Kandidaten weitgehend autonom gesteuerten, parteidistanten Kampagnen, die durch einen ausdifferenzierten Berufsstand professioneller Strategie- und Kommunikationsberater sowie Marketing- und Umfragespezialisten unterstützt werden. Diese wenden systematisch spezialisierte PR- und Publicity-Maßnahmen an, um ihre Themen und Deutungen auf die Medienagenda eines rein kommerziellen, strikt am Publikumsinteresse orientierten Fernsehsystems zu bekommen, dessen Nachrichtenredakteure von einem machtskeptischen, politikkritischen Rollenselbstverständnis geprägt sind.
} 
kampfführung und -wahrnehmung in den USA generell bestimme). Beide Positionen wurden von Esser und D'Angelo (2003; D'Angelo/Esser 2003) hinterfragt. Sie argumentierten erstens, dass Metaberichterstattung nicht andere Wahlthemen verdränge, sondern Themenverbindungen mit ihnen eingehe. Und sie argumentierten zweitens, dass Journalisten bei der Metaberichterstattung keineswegs auf eine uniforme Strategieorientierung festgelegt seien, sondern Medien und Publicity in verschiedenen Rollen präsentieren. Zu diesen Folgerungen kommen Esser und D'Angelo auf Basis ihres Framing-Ansatzes:

In Bezug auf ihr erstes Argument halten sie Framingstudien für besser geeignet, um Themen- und Frame-Verknüpfungen erkennen und erheben zu können. Weil bisherige Studien mit traditionellem Inhaltsanalyseansatz solche Verschmelzungen gar nicht erwarteten, fehlte ihnen auch der methodische Zugriff. Esser und D'Angelos Erwartung solcher Kombinationen fusst auf dem Konzept vom Interpenetrationscharakter mediatisierter Themen (Blumler/Gurevitch 1995: 26) und mediatisierter Ereignisse (Kepplinger 1990: 46, ff.), welches nahelegt, dass sich Medien- und Publicity-Thematisierung in erster Linie als Themenverflechtungen zeigen. So werden beispielsweise in einem Beitrag über "Medienkanzler Schröder" die Themen "Persönlichkeit/Charakter" und "Medien“ verwoben. In der Wahlberichterstattung sei nicht zu erwarten, dass Medien und Publicity als alleinige, isoliert stehende Beitragsthemen erscheinen; vielmehr stehen sie immer in Bezug zu einem Wahl(kampf)thema.

In Bezug auf ihr zweites Argument halten Esser und D'Angelo (2003) Framingstudien für besser geeignet, um die aktiven Strukturierungsprinzipien, die Journalisten bei der Framekonstruktion verwenden, theoriegeleitet nachzuvollziehen. So entwickeln sie theoretisch-deduktive Erwartungen darüber, dass Journalisten auch Alternativframes zum Strategieframe verwenden. Sie unterscheiden dabei Vermittlungs-, Strategie- und Verantwortlichkeitsframes. Die empirisch-induktive Bestätigung erfolgte mit dem von Gamson (1989) vorgeschlagenem Bottom UpVerfahren. Dieses leitet Frames aus den Texten empirisch her, indem die Framebestandteile von kleinsten über mittlere zu größeren Einheiten quantitativ erhoben werden (von Designatoren über Propositionen zu Skripts und schließlich Frames) und dann im Lichte der theoretischen Erwartungen verdichtend rekonstruiert werden. Der Mehrwert der Framinganalyse liegt darin, dass sie über das in traditionellen Inhaltsanalysen manchmal theorielose Erheben isolierter Texteinheiten hinausgeht, indem die kleinsten Codiereinheiten als Bausteine größerer bedeutungstragender Einheiten aufgefasst werden, welche auf Produktionsseite mit Schemata von Journalisten (oder anderen Kommunikatoren) und auf Wirkungs- 
seite mit Schemata von Rezipienten in Zusammenhang gebracht werden können, die alle auf derselben Operationaliserungsebene verortet sind.

Framingbasierte Inhaltsanalysen sind nach Überzeugung ihrer Befürworter besser in der Lage, latente Bedeutungen von Medientexten zu erfassen und theoriegeleitet zu erklären (Entman 1993: 57; Pan/Kosicki 1993: 58; Reese 2001: 8f.). Entsprechend ist zu fragen, was die theoretischen Ursachen für Metaberichterstattung sind. Die Nachrichtenforschung lässt erwarten, dass sich bei qualitativer Veränderung der Ereignislage und der journalistischen Arbeitsbedingungen auch die Qualität der Berichterstattung verändert. Ein Beispiel für die qualitative Veränderung der Ereignislage ist die Zunahme inszenierter oder mediatisierter Ereignisse, die von Publicity-Experten zum Zwecke der Berichterstattung initiiert oder kommunikationsstrategisch überformt werden. Diese strukturelle Mediatisierung wirkt reziprok auf die Berichterstattung zurück (Kepplinger 2001) und zeigt sich diskursiv in Metaberichterstattung. Wie lassen sich die verschiedenen Frames begründen?

Erklärung des Vermittlungsframes: Journalisten können bei ihrer Thematisierung der Rolle der Medien im Wahlkampf einen "Vermittlungsframe“ verwenden, wenn sie die Medien als Informationsvermittler präsentieren. Dies kann geschehen, indem sie die Präsenz von Kameras oder berichtenden Reportern bei einem Ereignis betonen oder den Umfang der Berichterstattung und das Ausmaß der Mediennutzung bei einem Thema beschreiben. Dass Journalisten überhaupt die Vermittlungsleistungen von Medien und Publicity im Wahlkampf hervorheben, erklären wir mit den international gültigen Professionsnormen der Genauigkeit und Vollständigkeit bei der Wiedergabe der vorgefundenen Realität (Nachrichtenund Professionstheorie). Dass sie darüber unter Akzentuierung der Vermittlungsfunktion berichten, erklären wir zum einen damit, dass dies der Primärfunktion von Medien und PR/Publicity in der Gesellschaft tatsächlich entspricht, zum anderen damit, dass sich Nachrichtenjournalisten zuvörderst als Vermittler verstehen (Funktions- und Rollenbildtheorie).

Erklärung des Strategieframes: Die Entwicklung, dass Medien und Publicity nicht nur als Vermittler, sondern auch als strategisches Instrument dienen, wird von den Journalisten mit "Strategieframes“ ausgedrückt. Im Laufe ihrer Erfahrung als Wahlkampfberichterstatter gewöhnen sich Journalisten an jene Machtspiele und Marketingüberlegungen, mit denen Politiker moderne Kampagnen führen (Habitualisierungstheorie). Weil Journalisten viel Zeit mit Kampagnenberatern und Kandidaten verbringen, lernen Journalisten, wie diese "Strategen" über die Rolle der Medien und der Publicity denken. Journalisten übernehmen diese strategische Denkweise in ihrer Berichterstattung (Lerntheorie). Von diesen Gesprächen lernen Journalisten auch, dass viele Politiker die Medien als eigensinnig, potent 
und konsequenzenreich wahrnehmen. Angesichts der zentralen Rolle, die die Nachrichtenmedien für den politischen Erfolg spielen, versuchen Politiker und Publicity-Experten die Berichterstattung in ihrem Sinne zu beeinflussen. Dadurch finden sich Journalisten häufig inmitten der Kampagnengefechte wieder, weil sich die Kontroll- und Lenkungsstrategien zur positiven Beeinflussung der Medienund Öffentlichkeitsagenda direkt auf ihre Arbeit beziehen. Anstatt sich diesen Nachrichtensteuerungsbemühungen zu unterwerfen, machen Journalisten diese Strategien in ihren Beiträgen selbst zu Thema. Dies kann einerseits als dekonstruierende Abwehrreaktion gegen Instrumentalisierungsversuche und andererseits als Demonstration der eigenen professionellen Autonomie gedeutet werden (Journalismus/PR-Konflikttheorie, Professionstheorie). Aber auch die kompetitive Marktzwänge setzen den Politikjournalismus unter Druck, ein möglichst spannendes, unterhaltendes Nachrichtenprodukt zu kreieren; dies ist mit einem Fokus auf wirkungsvolle oder fehlgeschlagenen Wettkampfstrategien eher zu realisieren als mit tiefschürfenden Problemdiskursen (Theorie der Aufmerksamkeitsökonomie). Erfahrene, und deswegen oft zynische, Journalisten vermuten hinter Politikeraktivitäten bald nur noch strategische Manöver zur Beeinflussung der Medien- und Öffentlichkeitsagenda; ähnlich wie sie werden auch ökonomisch denkende Journalisten das politisches Geschehen vornehmlich durch die Strategiebrille wahrnehmen. Gerade im Wahlkampf werden sie deswegen auch die Rolle der Medien und der Publicity mittels Strategieframes schildern.

Erklärung des Verantwortlichkeitsframes: Derart geprägte Metaberichterstattung lässt die Medien in einem reflektierten, analytischen, selbstkritischen Umgang mit ihrer gewachsenen Bedeutung erscheinen. "Verantwortlichkeitsframes" thematisieren die Medien als Instanz der Demokratie, die sich ihrer Sozialverantwortung bewusst, zur kritischen Auseinandersetzungen mit eigenen Fähigkeiten und Fehlleistungen in der Lage und zur Vermittlung von Medienkompetenz geeignet ist (Demokratie- und Sozialverantwortlichkeitstheorie). Neben dem demokratienormativen Bezug ist dieser Frame auch Ausdruck eines professionsinternen Qualitätsbewusstseins und Selbstkontrollmechanismus, indem die Vernachlässigung von Sorgfaltspflichten und Berichterstattungsnormen öffentlich kritisiert und berufsethische Grundsätze im Journalismus geschärft werden sollen (Professionstheorie). Im gleichen Maße will er die Rezipienten über die beruflichen Praktiken und Orientierungen von PR-und Publicity-Experten aufklären. Beim Framing der politischen Publicity werden instruktive, erhellende Informationen über den Politikvermittlungsprozess hervorgehoben, die den Wählern helfen sollen, realitätsgerechtere Vorstellungen über mediatisierte Politik zu entwickeln. Während hinter dem Vermittlungsframe das professionelle Selbstbild des Informationsjournalisten 
und hinter dem Strategieframe das professionelle Selbstbild des Kritikers steht, orientiert sich der Verantwortlichkeitsframe am Berufsverständnis des Erklärers und Wächters (Rollenbildtheorie). ${ }^{7}$

Innerhalb der vielfältigen Strömungen der Framingforschung ist die vorliegende Studie dem nachrichtenkonstruierenden, kommunikatorzentrierten FrameBuilding-Ansatz zuzurechnen (D'Angelo 2002: 877f., 880f.; Scheufele 1999: 115f.; Scheufele 2003: 49f., 93f.; Scheufele/Tewksbury 2007: 12f.). Die konkrete Umsetzung folgt dem „Model of Press and Publicity Framing“ (Esser/D'Angelo 2003: 623), für das zunächst die konstitutiven Bausteine von Frames zu bestimmen sind. Als kleinste verbale und visuelle Bausteine gelten Designatoren (auf Medien und Publicity verweisende Schlüsselwörter und -bilder), die einen Nachrichtenbeitrag mit einer Mindestintensität durchziehen müssen, um inhaltlicher Bestandteil des Themenprofils werden zu können. Designatoren als kleinste framing devices sind in Propositionen - bedeutungstragende Aussagen - eingebunden. Bei Propositionen handelt es sich um Behauptungen über die Wirklichkeit, die als Sprachakte entweder von Journalisten selbst oder aus von ihnen zitierten Quellen stammen. Mit der Analyse der Propositionen schreitet im Codierprozess die Frame-Bestimmung als Bottom-up-Verfahren von kleinsten zu größten Elementen voran. Es wurde bestimmt, ob die codierten Propositionen ausreichend diskursive Substanz darstellten, um zur Feststellung zu gelangen, dass der Text „über“ Medien oder Publicity handelte. Dabei qualifizierten sich nur solche Texte für die Frameanalyse, deren Propositionen quantitativ deutlich mehr als zehn Prozent aller Beitragsaussagen ausmachten und qualitativ die Codierereinschätzung bestätigen konnten, dass Medien oder Publicity mitbestimmender Teil des Themenprofils waren (Esser/D'Angelo 2003: 624-629). Im nächsten Schritt war zu bestimmen, ob die Propositionen ein klares Muster aufwiesen und sich in der Mehrzahl einer der oben ausgeführten Framecharakterisierungen zuordnen ließen. ${ }^{8}$ Im letzten Schritt wurden die Themenkombinationen erfasst, mit denen die Medien-Frames und PublicityFrames gemeinsam auftraten.

Journalisten konstruieren Frames durch die sinnstiftende, bedeutungsvolle Verbindung von Propositionen (Ereignisbeobachtungen, Quellenstatements, Eigenbewertungen), die einer im redaktionellen Sozialisierungsprozess erlernten Narrationsdramaturgie („Medienformate“) sowie den persönlich-professionellen Vermutungen über das abgelaufene Geschehenen („Hypothesen über die Reali-

\footnotetext{
7 Die theoretische Herleitung der Frames wurde stark beeinflusst durch die Arbeiten von Kerbel (1997, 1999), McNair (2000), McQuail (2005), Patterson (1993), Shoemaker/Reese (1996), Weaver (1998).

8 Zentrale Richtschnur bei dieser Zuordnung war die Codierung der Makropropositionen (Skripts), in die die Mikropropositionen (Aussagen) eingebettet waren. Sie spielen für die folgende Ergebnispräsentation jedoch keine Rolle.
} 
tät") des Verfassers folgen. Esser und D'Angelo (2003) fanden eine ausreichende Bandbreite der medien- und publicitybezogenen Propositionen in der amerikanischen Wahlkampfberichterstattung, die die Schlussfolgerung rechtfertigten, dass Journalisten die Rolle der Medien und der Publicity in drei Rollen wahrnehmen: einer Vermittlungsfunktion, einer Strategiefunktion, und einer Verantwortlichkeitsfunktion. In der theoretischen Herleitung dieser Frames lag der deduktive Ansatz, in der empirisch vorgefundenen Korrespondenz der verwendeten Propositionen der induktive Ansatz der Pilotstudie. Deren Ergebnisse sollen nun mit einem international vergleichenden Design auf ihre Generalisierbarkeit und Kontextabhängigkeit geprüft werden.

\section{Metaberichterstattung im Ländervergleich: Hypothesen}

In der vergleichenden politischen Kommunikationsforschung spielt es eine zentrale Rolle, wie der strukturelle Kontext des Politik- und Mediensystems jeweils den Untersuchungsgegenstand prägt. Dieser Festlegung liegt die Annahme zugrunde, dass unterschiedliche nationale Strukturen und Normen jeweils verschiedene politische Kommunikationsrollen und Handlungsweisen herausbilden bzw. unterdrücken. Daher werden komparative Untersuchungen häufig so angelegt, dass gezielt solche Länder ausgewählt werden, die sich hinsichtlich der Kontextbedingungen für das interessierende Phänomen unterscheiden (Pfetsch/Esser 2003). Basierend auf dieser Logik nimmt die vorliegende Studie an, dass unterschiedliche Ausprägungen der unabhängigen, erklärenden Variablen (also unterschiedliche Mediatisierungsgrade) in verschiedenen Systemkontexten korrespondieren mit unterschiedlichen Ausprägungen der abhängigen Variablen (also der Intensität und Frameverteilung von Metaberichterstattung). Solche quasi-experimentellen Forschungsdesigns verbieten eine streng kausale Ursachenattribution für die gefundene Varianz der abhängigen Variable. Eine „weiche Kontrolle“ der Varianz kann aber durch die systematische Kontextbeschreibung der institutionellen und kulturellen Besonderheiten erfolgen (Pfetsch/Esser 2003).

\subsection{Strukturelle Einflüsse auf Metaberichterstattung}

Entsprechend der unterschiedlichen Mediatisierungsgrade ihrer politischen Kommunikationssysteme wurden für die vorliegende Analyse die USA, Deutschland und Großbritannien ausgewählt. Der hohe strukturelle Mediatisierungsgrad in den 
USA ergibt sich zunächst aus dem institutionellen Kontext: Das präsidentielle Regierungssystem mit einem kandidatenzentrierten first past the post-Wahlsystem, einem durch primaries unterhöhlten Parteiensystem, einem durchkommerzialisierten Rundfunksystem und einem nur schwach regulierten Kampagnenreglement erlaubt in den USA viel personalisiertere, kapitalintensivere und vor allem medienbasiertere Wahlkämpfe als in Deutschland oder Großbritannien. Die vergleichsweise geringe Bedeutung der amerikanischen Parteien zeigt sich u.a. in der großen Zahl externer Kampagnenberater und Publicity-Experten, von denen im Präsidentschaftswahlkampf 2000 insgesamt 26 für George W. Bush und 21 für Al Gore arbeiteten (Dulio 2003). Dem gegenüber wurden im britischen Unterhauswahlkampf 2001 oder im deutschen Bundestagswahlkampf 2002 maximal je drei Werbe-, Umfrage- oder Media-Agenturen in den Kandidatenteams von Tony Blair, William Hague, Gerhard Schröder oder Edmund Stoiber gezählt (Althaus/Cecere 2003; Butler/Kavanagh 2002; D. Müller 2002; M. Müller 2002; Wring 2002).

Auch wurden mehr mediale Kommunikationskanäle im US-Präsidentschaftswahlkampf 2000 eingesetzt: Bush und Gore hielten drei live übertragene TVDuelle, während es in Deutschland nur zwei und in Großbritannien gar keine waren. Auch traten US-Kandidaten in verschiedenen TV-Unterhaltungsshows auf (u.a. Oprah Winfrey, Rosie O'Donnell, Jay Leno, David Letterman), wohingegen deutsche und britische Kandidaten auf diese Strategie wohl inszenierter Abendauftritte verzichteten. Auch TV-Werbung wurde in den USA sehr viel extensiver genutzt: Während des Vor- und Hauptwahlkampfes zwischen 1. Januar und 6. November 2000 wurden 302.450 Spots der Kandidaten und ihnen nahe stehender Gruppen ausgestrahlt (Goldman/Freedman 2002). Im deutschen Wahlkampf 2002 waren es dagegen nur rund 650 (Holtz-Bacha 2005; D. Müller 2002; M. Müller 2002) und in Großbritannien, wo bezahlte Fernsehwahlwerbung verboten ist, rund 100 Spots, für die den Parteien kostenlose Fernsehzeit zugewiesen wurde (Fisher 2001; Harrison 2002). Die Werbezentrierung amerikanischer Medienwahlkämpfe verschlingt enorme Summen. So gaben die Präsidentschaftskandidaten des Wahljahrs 2000607 Millionen Dollar für Werbe- und Publicity-Maßnahmen aus, und ihre Parteien weitere 692 Millionen Dollar in hard money and 498 Millionen Dollar in soft money (Nelson 2002). Dagegen lagen die Gesamtausgaben der britischen Parteien bei 37 Millionen Pfund und die der deutschen bei 81 Millionen Euro in den Wahljahren 2001 bzw. 2002 (Electoral Commission 2002; Hartmann et al. 2003).

Auch zwischen den beiden europäischen Ländern gab es wesentliche Unterschiede, die in den hier untersuchten Wahlkämpfen auf einen höheren Mediatisierungsgrad in Deutschland im Vergleich zu Großbritannien hinweisen. Von der seit längerem in Deutschland bestehenden Möglichkeit zum Kauf von Werbezeiten bei 
Privatsendern machten 2002 erstmals alle fünf der im 14. Deutschen Bundestag vertretenen Parteien Gebrauch; dagegen herrscht in Großbritannien breiter Konsens, den amerikanischen Weg der TV-Werbewahlkämpfe nicht einzuschlagen. (Holtz-Bacha 2005; Electoral Commission 2002). Ein weiteres amerikanisches Wahlkampfelement, dessen Einführung in Großbritannien bislang keine Chance hatte, erlebte im Bundestageswahlkampf 2002 seine Premiere: TV-Duelle. Eine dritte mediale Innovation in der deutschen Medienberichterstattung nach 1945 war die Wahlempfehlung (endorsement) einer nationalen Tageszeitung (der Financial Times Deutschland), die 2005 erhebliche Medienaufmerksamkeit erzielte. Wir erwarten folglich das höchste Niveau der Metaberichterstattung in den USA und das niedrigste in Großbritannien (Hypothese 1).

\subsection{Kulturelle Einflüsse auf Metaberichterstattung}

Das Niveau und Framing der Metaberichterstattung ist ebenfalls stark von kulturellen Faktoren abhängig. Politische und journalistische Kultur werden von Gurevitch und Blumler (2003: 383f.) als „Schlüssel“ zur Erklärung der Darstellungs- und Framingprozesse politischer Themen in der Nachrichtengebung bezeichnet. Im gleichen Sinne weist Pfetsch (2003: 395) darauf hin, dass die politische Kommunikationskultur „eine zentrale Rolle spielt für die Art und Weise, wie die öffentliche Thematisierung von Themen verläuft“. Die politische Kommunikationskultur, die den „Interaktionsraum an der Grenze zwischen Politik und Medien, in dem die Akteure der politischen Kommunikation sich bewegen" bezeichnet, sei in den USA viel stärker von der Medienlogik bestimmt als in Europa. Dies unterstreicht die in Hypothese 1 formulierte Annahme, erlaubt aber noch weitere wichtige Schlussfolgerungen.

Eine von der Medienlogik bestimmte Politikdarstellung lässt eine Wahlberichterstattung erwarten, die durch die Ziele und Kalküle der Journalisten und ihrer Medienorganisationen (im Gegensatz zu Politikern und ihren Parteiorganisationen) geprägt ist. ${ }^{9}$ Die Ziele und Kalküle der Journalisten im hochmediatisierten US-Wahlkampf beziehen sich erstens auf Distanzwahrung gegenüber Informa-

\footnotetext{
${ }^{9}$ In diesem Sinne meint „Mediatisierung der Berichterstattung“ die Prägung der Politikdarstellung nach medienspezifischen Selektions- und Präsentationsregeln (so Schulz 2003: 464f. mit Verweis auf den symbolischen Interaktionismus). Eine mediatisierte Politikdarstellung ist von der "discretionary power of the media" gekennzeichnet; sie ist geprägt von "the ability of journalists to tell the campaign story in their own words, scenarios, and assessments (rather than politicians'), to highlight the issues on which they believed the election should turn, and to initiate reports based on their news values and interests" (Blumler/Gurevitch 2001: 381)
} 
tionslenkungsbemühungen der politischen Publicity-Experten. Statt stenographischer Weiterleitung angedienter Botschaften streben Journalisten aus professionellem Eigeninteresse nach Autonomie und umfassender Kontrolle über den Thematisierungsprozess. Dieses Bestreben, ausschließlich medieneigene Nachrichtenwerte und Interpretationsmuster in der Berichterstattung zu berücksichtigen (und auf dieser Basis Framemodifikationen und -neukreationen oder Dethematisierungen und Themenausweitungen vorzunehmen) bringt Journalisten in Konflikt mit Politikern und ihren Publicity-Experten, die ebenfalls ein ausgeprägtes berufliches Interesse an Kommunikationskontrolle und Nachrichtensteuerung haben. Auf solche Einflussversuche reagieren politische Journalisten häufig mit einem professionellen Gegenimpuls, mit „counter-measures“ (Zaller 1999: 57) im Sinne eines „fight-back“ (Blumler 1997: 399). Die Ziele und Kalküle der Journalisten im hochmediatisierten US-Wahlkampf beziehen sich zweitens auf Publikumsorientierung und Profitorientierung (im Gegensatz zu Elitenrelevanz und Politisierung). Den Politiknachrichten wird der Stempel der Medienlogik aufgedrückt, um Lesern und Zuschauern ein möglichst attraktives, interessantes, marktgängiges Produkt bieten zu können.

Diese beiden Zielrichtungen des Journalismus, die hier als Ausprägungen einer spezifischen Kommunikationskultur beschrieben werden, ließen sich selbstverständlich ebenso als Ausprägungen einer spezifischen journalistischen Kultur oder politischen Kultur ansehen. Insgesamt lässt eine medienorientierte Kommunikationskultur ein Themenumfeld für Metaberichterstattung erwarten, das sich erstens auf Skandale, Fehlleistungen, Fehlurteile, Pannen, Entgleisungen oder Ausrutscher konzentriert (Skandal-Orientierung); zweitens auf Taktik, Image, Inszenierung, Stil und Siegeswillen fokussiert (Kampagnen-Orientierung); drittens die Politik sportlich dramatisiert durch den intensiven Einsatz selbstinitiierter Umfragen (Umfrage-Orientierung); viertens die Politik zu einem Wettstreit zwischen Stars personalisiert und dabei im Sinne einer Charakterpolizei die Persönlichkeit und das Persönliche vor dem Publikum ausbreitet (Personen-Orientierung); und fünftens die inhaltliche Substanz, programmatische Positionen und weltanschauliche Grundsatzfragen in den Hintergrund treten lässt (Inhalte- und Ideologie-Reduzierung).

Entsprechend des höheren Mediatisierungsniveaus erwarten wir den stärksten Trend zur Skandal-, Kampagnen-, Umfrage- und Personenorientierung in den USA und den schwächsten in den Großbritannien (Hypothese 2). Was die Metaberichterstattung selbst betrifft beklagt Vordenker Matthew Kerbel (1997; 1999), dass Medienselbstthematisierungen und Publicity-Thematisierungen zur Inhalte- und Ideologie-Reduzierung beitragen. Übertragen auf unsere Untersuchungsanlage, welche nicht von simplen Themenverdrängungen, sondern komplexen Themen- 
verknüpfungen ausgeht, operationalisieren wir seine Annahme folgendermaßen: Wir erwarten Kombinationen der Metaberichterstattung vor allem mit Skandal-, Kampagnen-, Umfrage und Personen-Themen und weniger mit Policy- und Ideologiethemen. (Hypothese 3).

Eine medienorientierte Kommunikationskultur befördert eine Art der Politikdarstellung, in der "the journalist, not the candidate or other newsmakers, is the primary communicator" (Hallin 1992: 11). Mit diesem "more journalist-centered“Stil sei meist auch eine Zunahme an strategy frames verbunden (Hallin 1992: 17f.). Weil sich Journalisten mit der Beurteilung substanzieller Politikinhalte überfordert fühlen, so Hallin, wählen sie die einfachere, schmackhaftere, leichter zu dramatisierende Variante der Beurteilung der Kampagnenführung. Dies geschehe mit strategy frames. Aus denselben Gründen erwartet Kerbel (1997), dass sämtliche Metaberichterstattung in einen Strategienebel („strategic haze") eingehüllt sei. Farnsworth und Lichter (2003: 79f., 105), die ebenfalls „media centred trends in coverage" untersuchten, fanden "self-referential coverage“ in den USA eng verknüpft mit Strategieorientierung. Entsprechend des höheren Mediatisierungsniveaus erwarten wir den größten Anteil an Strategieframes in der amerikanischen Metaberichterstattung (Hypothese 4). ${ }^{10}$ Aufgrund der Gemeinwohlverpflichtung von ARD und BBC erwarteten wir in der deutschen und britischen Metaberichterstattung mehr Verantwortlichkeitsframes. Abschließend wäre es von größtem Interesse zu sehen, welche charakteristischen Verknüpfungen es zwischen den Frames der Metaberichterstattung und den Themen der übrigen Wahlberichterstattung gibt (Forschungsfrage 1).

\subsection{Hypothesen im Überblick}

- H 1: Das Niveau der Metaberichterstattung ist in den amerikanischen Fernsehnachrichten am höchsten und in den britischen am niedrigsten.

- H 2: Das Themenumfeld der Metaberichterstattung ist in den amerikanischen Fernsehnachrichten stärker von einer Orientierung an Wahlkampf/Kampagnenführung, Umfragen/Bevölkerungsmeinung, Persönlichkeit/Charakter und Skandalisierung/Fehler sowie einer Dethematisierung von Inhalten/Positionen und Ideologie/Weltsicht geprägt als in den deutschen und britischen Fernsehnachrichten.

\footnotetext{
${ }^{10}$ Die bisherige Forschung zur deutschen und britischen Wahlkampfberichterstattung weist auf eine geringere Strategieorientierung - wenn auch bei zuletzt steigender Tendenz - hin (Semetko 2000: 372373; Blumler/Gurevitch 2001: 389; Genz et al. 2001: 409; Weiss 2005; Wilke/Reinemann 2001: 309).
} 
- $\quad$ H 3: Metaberichterstattung wird vor allem verknüpft mit Kampagnen-, Umfrage-, Personalisierungs- und Skandalisierungs-Themen.

- $\quad$ H 4: In der amerikanischen Metaberichterstattung überwiegen Strategieframes, in der deutschen und britischen Verantwortlichkeitsframes.

- Ff 1: Welche charakteristischen Verknüpfungen gibt es zwischen den Frames der Metaberichterstattung und den Themen der übrigen Wahlberichterstattung?

\section{$5 \quad$ Methode der Fernsehnachrichtenanalyse}

Bislang liegen systematische Vergleichstudien über amerikanische, britische und deutsche Wahlkampfberichterstattung kaum vor (zu den Ausnahmen gehören Semetko 1996 und Esser et al. 2001). Diese drei Länder wurden für die vorliegende Studie aufgrund der Logik des internationalen Vergleichs bewusst ausgewählt, weil sie sich hinsichtlich der theoretisch postulierten Bedingungsfaktoren für Metaberichterstattung unterscheiden. Als zentrale Bedingungsfaktoren werden struktureller Mediatisierungsgrad ${ }^{11}$ und medienorientierte Kommunikationskultur ${ }^{12}$ (als Konfigurationen unabhängiger Variablen) angenommen, die die Metaberichterstattung $^{13}$ (abhängige Variable) bestimmen. Mit solchen Designs erhofft man, die Verursachung und Kontextabhängigkeit eines Konzepts in verschiedenen nationalen Settings validieren sowie die transnationale Gültigkeit seiner theoretischen Prämissen generalisieren zu können (siehe Schaubild 1). ${ }^{14}$

\footnotetext{
${ }^{11}$ Operationalisiert über den Grad der Medien- und Publicity-Ressourcen im Umfeld der Akteure, Ereignisse, Tätigkeiten, Institutionen im politischen Kommunikationssystem zum Zeitpunkt der jeweiligen nationalen Wahl (USA 2000, UK 2001, D 2002); siehe Abschnitte 1 und 4.1 des Beitrags.

12 Operationalisiert über den Grad der Medienlogik als bestimmte Geschäftsgrundlage der Interaktionensbeziehungen und Handlungsorientierungen im politischen Kommunikationssystem zum Zeitpunkt der jeweiligen nationalen Wahl (USA 2000, UK 2001, D 2002); siehe Abschnitte 1 und 4.2 des Beitrags.

${ }^{13}$ Operationalisiert über die Intensität ihres Vorkommens und die Verteilung der drei unterschiedlichen Frames "Vermittlung", "strategischer Akteur" und "Verantwortlichkeit".

${ }^{14}$ Die bewusste Fallauswahl ist das entscheidende Kriterium der vergleichenden Methode. Sie übernimmt die Kontrollfunktion, die im Laborexperiment die bewusste und kontrollierte Manipulation der unabhängigen Variablen darstellt. Im vorliegenden Fall handelt es sich allerdings um eine Studie mit geringer Fallzahl (nur drei Länder), bei der die klassische Vergleichslogik der von Pzerworski und Teune (1970) entwickelten Most Similar Systems Designs oder Most Different Systems Designs nicht greift (vgl. Jahn 2006). Ich präsentiere hier ein Similar Systems With Different Outcomes (SS-DO)-Design, bei dem grundsätzlich eher ähnliche westliche Industriestaaten mit ähnlich stabilen politischen Kulturen verglichen werden, die laut theoretischer Erwartung unterschiedliche Intensitäten und Frameverteilungen der Metaberichterstattung zeigen (AV), weil sie sich in einigen entscheidenden struktu-
} 
Schaubild 1: Hypothesenleitendes Modell

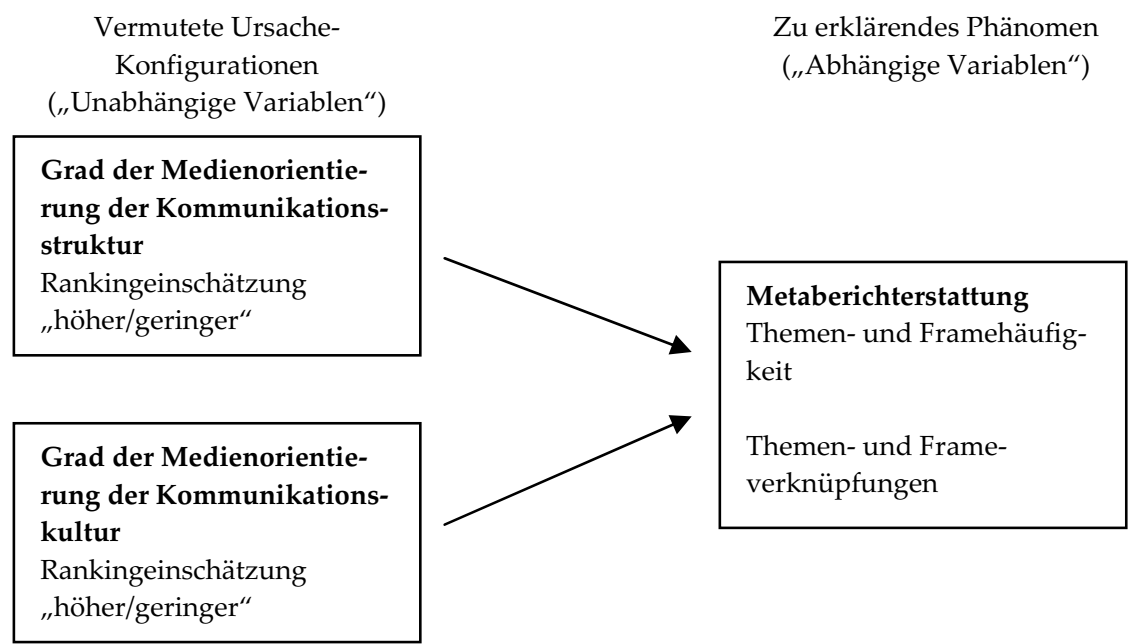

Unter Beachtung des Prinzips der Populationsäquivalenz wurden in allen drei Ländern die Flagschiff-Nachrichtensendungen der am meisten gesehenen Fernsehkanäle mit einer Vollerhebung für die Dauer der heißen Wahlkampfphase aufgezeichnet und von geschulten Codierern, zu denen auch der Autor als Projektleiter gehörte, mit einem detaillierten Codebuch analysiert (siehe Tabelle 1). ${ }^{15}$ Alle Sendungen nehmen einen prominenten 30minütigen Programmplatz ein und verfolgen einen einheitlichen, von "Anchors" moderierten Aufbau. Allerdings reduziert sich bei den kommerziellen Sendern ABC, NBC, ITV und RTL die Nettosendungsdauer um einige Werbeminuten. Ferner ist zu beachten, dass in Großbritannien der

rellen und kulturellen Bedingungsfaktoren unterscheiden (UVs). Der größte Schwachpunkt der SS-DO Strategie ist die positive Fallauswahl. Die Berücksichtigung weniger, als "geeignet" angesehener Fälle (USA, UK, D) mag für das konkrete Vorhaben forschungsökonomisch sinnvoll und gedankenlogisch illustrativ sein, aber unter methodologischen Aspekten ist eine möglichst große, zufällig zusammengesetzte Länderauswahl unbedingt vorzuziehen. Die einzige Rechtfertigung für SS-DO Designs ist die Verwendung einer originellen Theorie mittlerer Reichweite, die von Anfang an nur einen begrenzten Gültigkeitsbereich beansprucht und zur Generierung hypothesengeleiteter Forschung auf einem neuen Gebiet beitragen kann. Diese Sichtweise mache ich mir hier zu eigen und hoffe, mein Vorgehen damit rechtfertigen zu können.

${ }^{15}$ Für Unterstützungsleistungen bei diesem Projekt danke ich den Teilnehmern meines Post-Graduate Seminars "Quantitative Content Analysis" am Department of Communication der University of Oklahoma im Spring Term 2001, dem Political Communication Center an der University of Oklahoma, Lynda Lee Kaid von der University of Florida, Paul D'Angelo vom College of New Jersey, Margaret Scammell und Bernd Spanier an der London School of Economics and Political Science sowie Dominic Wring an der University of Loughborough. 
Untersuchungszeitraum halb so lange ist wie in den anderen beiden Ländern, weil die offizielle Wahlkampfphase dort auf vier Wochen festgelegt ist (Butler/Kavanagh 2002: 92; Semetko et al. 1991: 11). Wir folgten damit den Kampagnenperiodisierungen von Semetko (1996: 63), die in Deutschland und USA ebenfalls acht und in Großbritannien vier Wochen analysierte.

Tabelle 1: Inhaltsanalysedesign

\begin{tabular}{|c|c|c|}
\hline $\begin{array}{l}\text { US Präsidentschaftswahl- } \\
\text { kampf } \\
\text { Wahltag: 7. Nov. } 2000\end{array}$ & $\begin{array}{l}\text { UK Parlamentswahlkampf } \\
\text { Wahltag: 7. Juni } 2001\end{array}$ & $\begin{array}{l}\text { D Bundestagswahlkampf } \\
\text { Wahltag: 22. Sept. } 2002\end{array}$ \\
\hline $\begin{array}{l}\text { Analysezeitraum: } \\
\text { 9.9.-6.11.2000 }\end{array}$ & $\begin{array}{l}\text { Analysezeitraum: } \\
\text { 10.5. - 6.6.2001 }\end{array}$ & $\begin{array}{l}\text { Analysezeitraum: } \\
\text { 28.7. - 21.9.2002 }\end{array}$ \\
\hline $\begin{array}{l}\text { ABC World News Tonight (145 } \\
\text { Beiträge); NBC Nightly News } \\
\text { (139 Beiträge) - Vollerhebung }\end{array}$ & $\begin{array}{l}\text { BBC } 10 \text { O'Clock News (145 } \\
\text { Beiträge); ITV News at Ten (86 } \\
\text { Beiträge) - Vollerhebung }\end{array}$ & $\begin{array}{l}\text { ARD Tagesthemen (103 Beiträ- } \\
\text { ge); RTL Aktuell (71 Beiträge) - } \\
\text { Vollerhebung }\end{array}$ \\
\hline $\begin{array}{l}\text { Zuschauermarktanteil je Sen- } \\
\text { dung: ca. } 9 \%\end{array}$ & $\begin{array}{l}\text { Zuschauermarktanteil je Sen- } \\
\text { dung: ca. } 24 \%\end{array}$ & $\begin{array}{l}\text { Zuschauermarktanteil: ca. 12\% } \\
\text { (ARD-TT) bzw. 19\% (RTL-A) }\end{array}$ \\
\hline $\begin{array}{l}\text { Gesamt ( } 8 \text { Wochen): } \\
284 \text { Beiträge = 546 Minuten }\end{array}$ & $\begin{array}{l}\text { Gesamt ( } 4 \text { Wochen): } \\
231 \text { Beiträge = } 503 \text { Minuten }\end{array}$ & $\begin{array}{l}\text { Gesamt }(8 \text { Wochen }) \text { : } \\
174 \text { Beiträge }=479 \text { Minuten }\end{array}$ \\
\hline
\end{tabular}

In die Analyse gingen 284 amerikanische, 231 britische und 174 deutsche Beiträge ein. Die Berichterstattungsintensitäten waren sehr unterschiedlich: So wurden während der letzten zehn Werktage vor der Wahl 120 britische, 84 amerikanische und 48 deutsche Beiträge über den Wahlkampf ausgestrahlt. ${ }^{16}$ Die Erklärung für den niedrigen deutschen Wert liegt vermutlich bei einem bereits früher entdeckten grundlegenden Auffassungsunterschied über professionelle Nachrichtengebung: Während amerikanische und britische Redakteure dem Wahlkampf einen generellen Nachrichtenbonus verleihen, der breite Informierung ermöglichen und den

${ }^{16}$ Die Beschränkung auf die „letzten zehn Werktage vor der Wahl“ wurde aus Gründen der Vergleichbarkeit gewählt. Hier einige wichtige Hintergrundinformationen: Die Gesamtzahl der britischen Meldungen liegt in Wahrheit höher als in Tabelle 1 angegeben, weil aus technischen Gründen drei Werktagsnachrichtensendungen sowie grundsätzlich die Wochenendnachrichtensendungen von BBC und ITV nicht aufgezeichnet werden konnten; für die USA und Deutschland gingen die Samstags- und Sonntagsausgaben hingegen in die Analyse ein. Zur Einordnung des niedrigen deutschen Wertes sei auch an die so genannte "Jahrhundertflut" erinnert, die dazu führte, dass Mitte August acht Tage lang kein einziger wahlkampfrelevanter Aspekt in Tagesthemen und RTL-Aktuell thematisiert wurde. $\mathrm{Zu}$ Großbritannien ist der Vollständigkeit halber anzumerken, dass aufgrund eines Ausbruchs der gefährlichen Maul- und Klauenseuche im Frühjahr 2001 der Ausnahmezustand verhängt und der ursprüngliche Wahltermin vom 3. Mai auf den 7. Juni verschoben wurde (Butler/Kavanagh 2002) 
sonst üblichen Präsenznachteil der Oppositionsparteien auf dem Bildschirm aufwiegen soll, lassen sich deutsche Redakteure geradezu demonstrativ von Wahlkampfaktivitäten nicht beeindrucken und bestehen auf Auswahlkriterien strikt nach Nachrichtenwert, was zur Ignorierung vieler Kampagnenereignisse führt (Semetko 1996; Genz et al. 2001). Deutsche Redakteure überlassen die nicht-tagesaktuellen, nicht-neuigkeitsorientierten Wahlkampfaspekte eher den zahlreichen Sondersendungen (Zubayr/Gerhard 2002), denen amerikanische Networks kaum Raum gewähren (Farnsworth/Lichter 2003). Die britischen Programme bieten Wahlkampfschwerpunkte sowohl innerhalb ihrer Nachrichtensendungen als auch in zusätzlichen Sondersendungen (Harrison 2002).

Frame wird definiert als ,a central organizing idea for news content that supplies a context and suggests what the issue is through the use of selection, emphasis, exclusion, and elaboration“ (Tankard 2001: 100f.). Die beiden zentralen "issues“ dieser Studie sind Medien und Publicity, welche mittels "selection, emphasis, exclusion, and elaboration" in drei Rollen präsentiert werden: Vermittlung, Strategie und Verantwortlichkeit. Entsprechend dieser unterschiedlichen Einbettungen werden unterschiedliche Deutungen, Kausalinterpretationen und Bewertungen beim Rezipienten evoziert (so Entman 1993: 52 in seiner berühmten Framingdefinition). Die Erfassung der Sonderthemen Medien und Publicity sowie der acht übrigen Themen Wahlkampf/Kampagne, Umfragen/Bevölkerungsmeinung, Persönlichkeit/Charakter, Skandalisierung/Fehler, Inhalte/Positionen, Kandidatenbewertung, Ideologie/Weltsicht sowie Politiksystem/Wahlsystem erfolgt mit einem an Kerbel et al. (2000) angelehnten Klassifikationsschema, wobei Modifikationen gemäß der neu gewählten Ländervergleichsperspektive und der neu gewählten Framing-Konzeption erforderlich wurden.

\section{Ergebnisse}

\subsection{Erste Hypothese: Intensität der Metaberichterstattung}

Die erste theoretische Erwartung, wonach das Niveau der Metaberichterstattung am höchsten in den USA und am niedrigsten in Großbritannien ist, wird durch die empirische Analyse der Fernsehhauptnachrichtensendungen bestätigt. Eine Auswertung der verbalen und visuellen Designatoren zeigt, dass 41\% der analysierten amerikanischen Wahlkampfbeiträge einen "gemäßigten“ oder „hohen“ Anteil an Schlüsselwörtern oder Schlüsselbildern, die auf Medien- oder Publicity-Themati- 
sierungen hindeuten, aufweisen. ${ }^{17}$ In Deutschland sind es 38\% und in Großbritannien $14,5 \%$ (siehe Tabelle 2). Die Unterschiede zwischen amerikanischem und britischem Metaberichterstattungsniveau sind extrem robust (Cramer's V = .29; n = 515 Beiträge), die zwischen amerikanischem und deutschem dagegen statistisch unerheblich (Cramer's V = .02; $\mathrm{n}=458$ Beiträge). ${ }^{18}$

Tabelle 2: Intensität der Metaberichterstattung

\begin{tabular}{lccc}
\hline & $\begin{array}{c}\text { USA } \\
(\mathrm{N}=284 \text { Beiträge })\end{array}$ & $\begin{array}{c}\text { UK } \\
(\mathrm{N}=231 \text { Beiträge })\end{array}$ & $\begin{array}{c}\text { D } \\
(\mathrm{N}=174 \text { Beiträge })\end{array}$ \\
\hline $\begin{array}{l}\text { Beiträge mit Medien- } \\
\text { Thematisierung }{ }^{a}\end{array}$ & $14,5 \%$ & $11 \%$ & $24 \%$ \\
\hline $\begin{array}{l}\text { Beiträge mit Publicity- } \\
\text { Thematisierung }{ }^{b}\end{array}$ & $21,5 \%$ & $3,5 \%$ & $6 \%$ \\
\hline $\begin{array}{l}\text { Beiträge mit Medien- und } \\
\text { Publicity-Thematisierungen }{ }^{a, b}\end{array}$ & $5 \%$ & $0 \%$ & $8 \%$ \\
\hline $\begin{array}{l}\text { Total: Beiträge mit Meta- } \\
\text { berichterstattung }{ }^{a, b}\end{array}$ & $\mathbf{4 1 \%}$ & $\mathbf{1 4 , 5 \%}$ & $\mathbf{3 8 \%}$ \\
\hline $\begin{array}{l}\text { Restsumme: Beiträge ohne substan- } \\
\text { tielle Metaberichterstattung }\end{array}$ & $59 \%$ & $85,5 \%$ & $62 \%$ \\
\hline
\end{tabular}

a Gemessen über verbale oder visuelle Designatoren, die auf Presse, Fernsehen, Studio, Kamera, Artikel, Berichterstattungsserien, Reporter, Medien, Namen von Zeitungen oder Sendungen usw. auf zumindest "gemässigtem" Niveau verweisen.

b Gemessen über verbale oder visuelle Designatoren, die auf Maßnahmen des Kommunikationsmanagements, Werbung, Image, Kampagnen- und Medienberater, inszenierte Auftritte usw. auf zumindest "gemässigtem" Niveau verweisen.

Zwei Aspekte sind auffällig: Zum ersten werden in Deutschland und Großbritannien politische Publicity-Maßnahmen seltener thematisiert als in den USA $(6 \%$ bzw. 3,5\% gegenüber 21,5\%), was auf einen unvermindert geringeren Professiona-

\footnotetext{
17 Designatoren weisen zunächst auf Medien- und Publicity-Thematisierungen hin (ihr Beitrag als kleinste Bausteine für Frames wird später analysiert). Waren sie in mehr als 50\% des Berichtes präsent, wurde ein "hohes" Designatorenniveau codiert, bei 10 bis 50\% ein "gemäßigtes" Niveau. Für eine weitere Framing-Analyse kommen nur Beiträge mit einem zumindest "gemäßigtem“ Medien- und Publicity-Designatorenniveau in Frage.

${ }^{18}$ Die Korrelationsberechnung bezieht sich auf die Verteilung in den Zeilen ,Zwischensumme' und ,Restsumme' von Tabelle 2. Die Ausweisung von statistischen Signifikanzwerten erscheint wegen der zugrunde liegenden Vollerhebung nicht angebracht. Sie ist auch deswegen nicht angebracht, weil es sich bei den analysierten Medien nicht um eine zufällige, sondern um eine bewusste Auswahl (von Leitmedien) handelt.
} 
lisierungsgrad auf diesem Gebiet in Europa hindeutet. Zum zweiten thematisierten die deutschen Fernsehnachrichten ungewöhnlich stark die Rolle und Präsenz der Nachrichtenmedien im Wahlkampf $(24 \%+8 \%=32 \%)$. Das spricht dafür, dass (a) das politische Kommunikationssystem durch den Bundestagswahlkampf 2002 einen erheblichen Mediatisierungsschub erfahren hat, dass (b) dieser Mediatisierungsschub vor allem mit der gewachsenen Rolle der Nachrichtenmedien zu tun hat und dass (c) die Nachrichtenmedien diesen Bedeutungszuwachs selbst intensiv thematisiert haben. In der Tat wurden erstmals nach amerikanischem Vorbild zwei Kandidatendebatten im Fernsehen abgehalten, obwohl das komplizierte deutsche Koalitionssystem solche zugespitzten Zweierduelle eigentlich unmöglich machen sollte. Dabei waren ARD und RTL von dem Erfolg, den Wahlkampf zunehmend in ihre Fernsehstudios zu verlagern, so eingenommen, dass sie die Kandidatendebatten als „Duelle“ überhöhten und wie ein Formel 1-Rennen oder eine Skisprungmeisterschaft antrailerten. Weiterhin hat mit der Financial Times Deutschland erstmals eine deutsche Tageszeitung nach amerikanischem Vorbild eine begründete Wahlempfehlung abgegeben (die New York Times macht dies seit 1852), obwohl dies als eine für Deutschland unangemessene „Bevormundung der Leser" und als ein fragwürdiger "Schritt auf dem Weg der Amerikanisierung“ kritisiert wurde (so Siegfried Weischenberg gegenüber $d p a$ am 16.9.2002). Die Zeitung selbst begründete ihren „bewussten Tabubruch" damit, dass es nach angelsächsischer Tradition bei wichtigen Ereignissen wie einer Parlamentswahl zur „wesentlichen Aufgabe“ einer Zeitung gehöre, mit offenem Visier „vorher Stellung zu beziehen“ (FTD vom 16. September 2002). Die Entwicklung der deutschen Medien zu einem politischen Akteur - dem nach verbreiteter Auffassung die hiesigen Systemstrukturen entgegenstehen müssten - beklagte auch das Kanzlerehepaar Schröder, als es die BildBerichterstattung über die private Nutzung dienstlich erflogener Bonusmeilen durch Regierungspolitiker als Kampagne gegen Rot-Grün und parteipolitische Einmischung eines mächtigen Blattes in den Wahlkampf bezeichnete (Berg 2002).

\subsection{Zweite Hypothese: Themenumfeld der Metaberichterstattung}

Metaberichterstattung ist Teil der generellen Wahlberichterstattung. Hinsichtlich der generellen Wahlberichterstattung lässt eine stark medienorientierte Kommunikationskultur und -struktur eine Nachrichtengebung erwarten, die weniger an der Wiedergabe von Informationsangeboten der Kandidaten und ihrer Publicity-Experten interessiert ist und mehr an einer eigenständigen Intervention in den Themensetzungsprozess. Entsprechend erwartet Hypothese 2 ein Themenumfeld der 
Metaberichterstattung, das vor allem in den USA von einer deutlichen Orientierung an Wahlkampf/Kampagnenführung, Umfragen/Bevölkerungsmeinung, Persönlichkeit/Charakter und Skandalisierung/Fehler sowie einer Dethematisierung von Inhalte/Positionen und Ideologie/Weltsicht gekennzeichnet ist.

Zur Überprüfung wurden für jeden Beitrag - zusätzlich zu den Medien- und Publicity-Thematisierungen - die sonstigen Themen erfasst. ${ }^{19}$ Das sich daraus ergebende Themenprofil der amerikanischen, britischen und deutschen Vorwahlberichterstattung weist ein verblüffend einheitliches Muster auf (siehe Tabelle 3). Die

Tabelle 3: Themenprofil der Wahlberichterstattung im Umfeld der Metaberichterstattung

\begin{tabular}{|c|c|c|c|c|c|}
\hline \multirow{2}{*}{\multicolumn{2}{|c|}{ Wahlkampfthemen }} & $\begin{array}{c}\text { USA } \\
(\mathrm{N}=284 \\
\text { Beiträge })\end{array}$ & $\begin{array}{c}\text { UK } \\
(\mathrm{N}=231 \\
\text { Beiträge })\end{array}$ & $\begin{array}{c}\text { D } \\
(\mathrm{N}=174 \\
\text { Beiträge })\end{array}$ & \multirow{2}{*}{$\begin{array}{c}\text { Rangfolge } \\
\text { der meist- } \\
\text { berichteten } \\
\text { Themen in } \\
\text { allen drei } \\
\text { Ländern }\end{array}$} \\
\hline & & \multicolumn{3}{|c|}{$\begin{array}{c}\text { Anteil der Beiträge, die das } \\
\text { Wahlkampfthema enthalten (\%) }\end{array}$} & \\
\hline \multicolumn{6}{|l|}{ Polity } \\
\hline & Wahlsystem/Politisches System & 6 & 4 & 6 & \\
\hline \multicolumn{6}{|l|}{ Policy } \\
\hline & Ideologie/Weltsicht & 2 & 2 & 1 & \\
\hline & Kandidaturbewertung & 8 & 5 & 3 & \\
\hline & Inhalte/Positionen & 36 & 43 & 36 & (2.) \\
\hline \multicolumn{6}{|c|}{ Personality } \\
\hline & Persönlichkeit/Charakter & 19 & 9 & 13 & (4.) \\
\hline & Skandalisierung/Fehler & 4 & 6 & 13 & \\
\hline \multicolumn{6}{|c|}{ Politics/Process } \\
\hline & Wahlkampf/Kampagne & 57 & 65 & 57 & (1.) \\
\hline & Umfragen/Bevölkerungsmeinung & 35 & 15 & 26 & (3.) \\
\hline Total ${ }^{a}$ & & $167 \%$ & $149 \%$ & $155 \%$ & \\
\hline
\end{tabular}

a Total ist höher als $100 \%$ weil pro Beitrag bis zwei Themen erfasst wurden. In den 284 US-Beiträgen gab es 472 Themennennungen $(=167 \%)$, in den 231 UK-Beiträgen 350 Themennennungen $(=149 \%)$, und in den 174 deutschen Beiträgen 269 Themennennungen (=155\%).

\footnotetext{
${ }^{19}$ Korrespondierend zum Vorgehen bei der Erhebung der Themen Medien und Publicity wurde auch bei den weiteren Wahlkampfthemen zwischen Erstthema (das mehr als 50\% des Beitragsinhalts beansprucht), Zweitthema (das 10-50\% beansprucht) und Randthema (das unter 10\% beansprucht) unterschieden. Pro Beitrag wurden bis zu drei Themen codiert, die vorliegende Auswertung beruht jedoch nur auf einer Betrachtung der Erst- und Zweitthemen und blendet Randthemen völlig aus.
} 
Schwerpunktsetzung der amerikanischen Nachrichtengebung korreliert in höchstem Maße mit der deutschen (Spearman's rho $=.87$ ) und britischen (Spearman's rho $=.93$ ), was als anschaulicher Beleg für transnationale Konvergenztendenzen in der Wahlberichterstattung gedeutet werden kann. ${ }^{20}$ Die acht Einzelthemen entstammen vier Bereichen: Policy, Politics/Process, Personality, Polity. Nach den Prämissen der normativen Demokratietheorie ist eine intensive Informierung der Bürger über Policy-Aspekte von höherer Bedeutung für informierte Wahlentscheidungen als über Politics/Process- oder Personality-Aspekte. Allerdings legen die Nachrichtensendungen aller drei Länder ihren Berichterstattungsschwerpunkt auf das Politics/Process-Thema ,Wahlkampf/Kampagne', das über Taktiken, Techniken, Personen und Organisationen der Kampagnenführung zur Erreichung des Wahlsiegs informiert. Diese Präferenz drückt sich in den britischen Daten noch etwas stärker aus (65\% gegenüber 57\%), weil die zeitliche Beschränkung der Kampagne auf vier Wochen dem Stimmenwettbewerb einen besonders intensiven Charakter verleiht. Das zweite Politics/Process-Thema ,Umfragen/Bevölkerungsmeinung' bestätigt die häufig beschriebene Horse-Race-Fixierung amerikanischer Nachrichten, die mittels selbstinitiierter Umfragen den Wahlkampf als sportliches Kopf-an-Kopf-Duell anheizen (35\%). Die Präferenz für umfragegestützte Spekulationen darüber, welcher Kandidat und welcher Politikvorschlag öffentliche Unterstützung gewinnt oder verliert, war im Bundestagswahlkampf 2002 auch in Deutschland hoch (26\%). ARD und RTL hatten nach amerikanischem Vorbild Verträge mit Umfrageinstituten geschlossen, um regelmäßig die selbst bestellten Daten als Exklusivnachrichten vorstellen zu können.

Gegenüber den Politics/Process-Aspekten nahmen die Policy-Aspekte in allen drei Ländern einen geringeren Raum ein. Ein Drittel der amerikanischen und deutschen (36\%) und fast die Hälfte der britischen Wahlkampfbeiträge (43\%) boten unter dem Thema ,Inhalte/Positionen' ihren Zuschauern substanzielle Informationen über Programme, Positionen, Politikfelder, Probleme und Lösungsvorschläge. Ein weiterer wichtiger Policy-Aspekte lässt sich als prospektive und retrospektive ,Kandidaturbewertungen' bezeichnen und bietet substanzbasierte Einschätzungen hinsichtlich der Erfahrungen, Fähigkeiten, Erfolge und Misserfolge der Kandidaten in der Vergangenheit und ihrer vermutlichen zukünftigen Schwerpunktsetzung und Kursrichtung. Solche für Entscheidungsprozesse wichtige Bestandsaufnahmen spielen in der Vorwahlberichterstattung der drei Länder ebenso wenig eine zentrale Rolle (3-8\%) wie Informationen über das Policy-Thema ,Ideologie/Weltsicht' (1-2\%). Hiermit ist übergeordnetes Orientierungswissen im Sinne einer Kontext-

${ }^{20}$ Auf die Ausweisung von Signifikanzwerten wird aus vorgenannten Gründen durchgehend verzichtet. 
einordnung der Ideen, Werte, Überzeugungen und Philosophien der Kandidaten in Kategorien der politischen Weltanschauung gemeint. Solche Grundsatzfragen, in denen die Wahl als Alternative zwischen politischen Weltbildern diskutiert wird, scheinen in modernen Kampagnen(-nachrichten) keine Rolle mehr zu spielen.

Deutlich größere Beachtung erlangen dagegen in allen Nachrichtensendungen die Personality-Nachrichten. Beim Thema ,Persönlichkeit/Charakter' werden privates Umfeld, persönliche Eigenschaften und Wesensart sowie Züge des Charakters und der Persönlichkeit berichtet. Dies spielt in den USA aufgrund der kandidatenorientierten Wahlkampfführung eine große Rolle (19\%), erlangte aber in auch Deutschland - angeregt durch die erstmaligen Kandidatenduelle - höhere Aufmerksamkeit (13\%). Beim zweiten Personality-Thema ,Skandalisierung/Fehler' geht es um angeprangertes Verhalten von Politikern, negative Enthüllungen, öffentliche Ausrutscher, schädliche Gerüchte oder witzhafte Bloßstellungen. Hier nehmen die deutschen Nachrichten eine führende Stellung ein (13\%), weil sie u.a. Vorwürfen gegen die private Bonusmeilennutzung von Parlamentariern (Cem Özdemir und Gregor Gysi traten zurück), gegen israelkritische Aussagen von NRW-Wahlkämpfer Möllemann sowie gegen einen vermeintlichen Bush-HitlerVergleich von Justizministerin Däubler-Gmelin breiten Raum einräumten. Diese Vorwürfe beschäftigen Tagesthemen und RTL-Aktuell doppelt so intensiv wie das Polity-Thema ,Politisches Wahlsystem' (6\%), bei dem der institutionell-regulative Rahmen des Politik- und Wahlsystems erklärt wird und der Zuschauer u.a. über Wahlregeln, Wahlbeteiligung, politische Institutionen, Hintergründe und Partizipationsmöglichkeiten informiert wird.

Zusammenfassend lässt sich feststellen, dass deutsche und britische Wahlberichterstattung der amerikanischen in Themenstruktur und Schwerpunktsetzung in höchstem Maße entsprechen. Bei den europäischen Ländern findet sich - von graduellen Unterschieden abgesehen - eine vergleichbare Orientierung an Skandalisierungen, Kampagnenführung, Umfragen und Persönlichkeiten. Damit ist Hypothese 2 für die hier untersuchten Wahlkämpfe widerlegt. Falls die hier präsentierten Querschnittdaten auf einen Längsschnitttrend hindeuten sollten, müsste man folgern, dass die Unterschiede in den Strukturen und Kulturen der untersuchten politischen Kommunikationssystemen nur noch als schwache Bremsen auf dem Weg zu einem transnationalen Berichterstattungskurs wirken. Anders formuliert: Trotz institutioneller und struktureller Unterschiede gleichen sich die handlungsleitenden Kulturen an. Entsprechende Hinweise auf eine Annäherung der Kommunikationskulturen fand auch Pfetsch (2001: 35). Sie entdeckte eine wachsende „Ähnlichkeit der Orientierungen der deutschen Journalisten mit den 
US-amerikanischen Akteuren“, sodass „man fast schon von einer ,Amerikanisierung' der deutschen Korrespondenten sprechen" könne.

Insgesamt erscheint es jedoch angemessener, statt von Amerikanisierung besser von Hybridisierung zu sprechen: Traditionelle, nationenspezifische Medienkulturen bleiben (in Deutschland und Großbritannien) erhalten, werden allerdings immer stärker vom transnationalen Einfluss einer autonom-kommerziellen Medienlogik überlagert (die in den USA am längsten etabliert und am offensivsten transportiert wird), was u.a. auch zu Angleichungstendenzen im Themenprofil bei der Berichterstattung doch eigentlich recht unterschiedlicher Wahlkämpfe führen kann. Dafür spricht auch der in weiteren - hier nicht dargestellten - Auswertungsschritten erkannte Profilverlust von ARD und BBC, denn die Themenstruktur der öffentlich-rechtlichen Sender korreliert hochgradig mit der der privat-kommerziellen Konkurrenz. In Deutschland und Großbritannien scheint nicht mehr die Orientierung am Gemeinwohl, sondern am Publikumserfolg bestimmend zu werden. Allerdings werden erst Langzeitstudien zeigen können, ob sich diese Tendenzen verallgemeinern lassen.

\subsection{Dritte Hypothese: Themenverknüpfung zwischen Metaberichterstattung und übriger Wahlberichterstattung}

Die dritte Hypothese, dass Metaberichterstattung diskursive Verwandtschaft mit Personality- und Politics/Process-Themen aufweist, bestätigt sich für alle drei untersuchten Länder. Wie von Kerbel vermutet, werden Medienselbstthematisierungen und Publicity-Thematisierungen besonders häufig verknüpft mit den Themen Skandalisierung/Fehler, Persönlichkeit/Charakter, Wahlkampf/Kampagne und Umfragen/Bevölkerungsmeinung (siehe Tabelle 4), wohingegen dies mit den Themen Inhalte/Positionen, Kandidaturbewertungen, Ideologie/Weltsicht und Politik-/ Wahlsystem kaum vorkommt. Eine Ausnahme, die allerdings nur auf einem einzigen Fall beruht, stellt Ideologie/Weltsicht in Deutschland dar. ${ }^{21}$ Schließt man diesen Sonderfall für einen Augenblick aus und überprüft das Themenverknüpfungsprofil der drei Länder auf ihre statistische Ähnlichkeit, bestätigen hochrangige

\footnotetext{
${ }^{21}$ Am 16. September 2002 berichteten die ARD-Tagesthemen über die Wahlempfehlung der FTD und gaben dem redaktionsinternen Entscheidungsprozess breiten Raum, der nach sechs Stunden Redaktionskonferenz zum Ergebnis kam: Am besten sind die ,Grünen', aber weil es die nur mit den verbrauchten ,Roten' gibt, ist es Zeit für einen Wechsel zu den ,Schwarzen'. Dieser Beitrag war der einzige, der die Bundestagswahl tatsächlich als Alternative zwischen unterschiedlichen ideologischen Richtungsangeboten darstellte. Durch seine Einbettung in die FTD-Redaktionsdebatte (bei der laut ARDTagesthemen mehrere Fernsehteams anwesend waren) erhielt er eine Medien-Verknüpfung.
} 
Korrelationswerte die enge Übereinstimmung zwischen den drei Ländern (Spearman's rho für D/UK ist .83; für D/USA rho=.90; für UK/USA rho=.92). Hinsichtlich der Theoriebildung lässt sich folgern, dass Metaberichterstattung - unabhängig von institutionellen und strukturellen Unterschieden - länderübergreifend vor allem an Personality- und Politics/Process-Themen gebunden ist. Besonders erwähnenswert ist die auffallend enge Verknüpfung von Metaberichterstattung mit Skandalberichterstattung (Tabelle 4). Skandalisierungen scheinen vor allem mediengetriebene Phänomene zu sein, die bei den betroffenen Politikern PublicityReaktionen auslösen, welche wiederum zum Gegenstand der Berichterstattung werden.

\subsection{Vierte Hypothese: Frames der Metaberichterstattung}

Im Sinne des von Gamson (1989) propagierten Verständnisses von Framing als „value added process" begreifen wir Medien-Frames und Publicity-Frames als originär journalistische Erweiterung (oder Komplettierung) der Politikdarstellung um Interpretationskontexte, die den mediatisierten Charakter der Wahlkampagnen auszudrücken. Wir sehen diese Frames als eine plausible narrative Form, um diesem komplizierten Mediated Politics-Umfeld als Berichterstatter gerecht zu werden. Diese Sichtweise ist weit offener als Kerbels beschränkte Erwartung, dass jegliches Medien- und Publicity-Framing strategisch motiviert sei. Wir halten ein Medienund Publicity-Framing aus Vermittlungs- und Verantwortungsperspektive für genau so erwartbar - wobei Hypothese 4 aufgrund des höheren Mediatisierungsniveaus eine stärkere Strategiefixierung für die USA vorhersagt.

Wie Tabelle 5 zeigt (siehe Spalte ,Gesamt'), werden die Medien vornehmlich in ihrer Vermittlerrolle angesprochen, deren Funktion in der neutralen Verbreitung und Weiterleitung von Informationen liegt. Deutlich seltener werden sie als strategische Mitakteure präsentiert, von denen eigenständige Resonanzen ausgehen, die ins Kalkül der übrigen Akteure eimbezogen werden müssen. Das Bild ändert sich, wenn wir uns der Publicity-Seite zuwenden. Aspekte des Themen-, Image-, Ereignis- und Newsmanagements sowie des Marketings und der Werbung von den Kandidaten und ihren Beratern werden bevorzugt als von taktischem Kalkül motiviert dargestellt - mittels strategischer Publicityframes. Das Gesamtbild, wonach insgesamt etwas mehr Vermittlungsframes als Strategieframes verwendet werden und insbesondere die Medien eher mit einer Vermittlungs- als mit einer Strategieorientierung dargestellt werden, zeigt sich für alle drei untersuchten Ländern gleichermaßen. Ebenfalls in allen Ländern gleichermaßen erkennbar ist eine nur gerin- 
ge Bedeutung der Verantwortlichkeitsframes, welche üblicherweise auf eine reflektierende Auseinandersetzung mit dem demokratischen Auftrag und den professionellen Berichterstattungsnormen der Medien hindeuten bzw. sich um Medienaufklärung und Publicity-Hinterfragung bemühen. Zwar ist die Strategieorientierung der amerikanischen Fernsehnachrichten etwas ausgeprägter, aber der Unterschied zu den europäischen Fernsehnachrichten fällt schwächer aus als es die bisherigen Erkenntnisse zu den Kommunikationsstrukturen und -kulturen erwarten ließen. Die nur moderate Bestätigung für Hypothese 4 ist ein weiterer Hinweis auf

Tabelle 4: $\quad$ Charakteristische Kombinationen von Medien-/Publicity-Themen mit übrigen Wahlkampfthemen

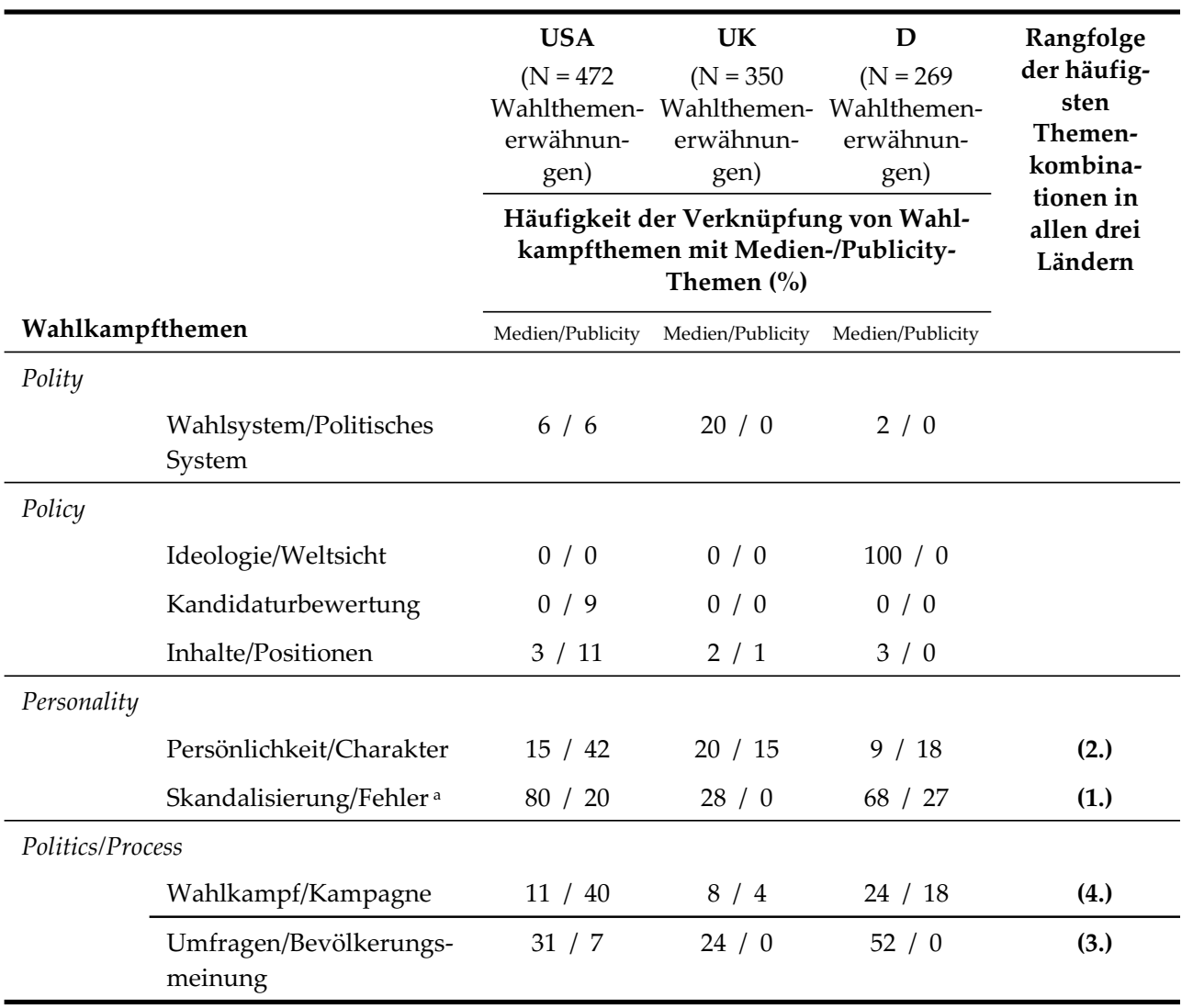

a Lesebeispiel: In 80\% der Fälle, in denen die US-Nachrichten einen Politiker-Skandal oder -Fehler thematisierten, wurde dabei auch die Rolle der Medien thematisiert; in 20\% der Fälle wurde dabei auch die Rolle von Publicity-Maßnahmen thematisiert. In den britischen Fernsehnachrichten wurden Skandalisierungen/Fehler dagegen viel seltener im Kontext von Medien (28\%) oder Publicity (0\%) berichtet. 
Angleichungstendenzen, die im bisherigen Forschungsstand noch hinreichend akzentuiert worden waren. Andererseits verweisen sie aber auch - im Sinne der Konzeptverifikation - auf eine länderübergreifende Verallgemeinerung wesentlicher Prämissen der Metaberichterstattungsforschung.

Tabelle 5: Verteilung von Vermittlungs-, Strategie-, und Verantwortlichkeitsframes in Prozent (\%)

\begin{tabular}{lcccc}
\hline & $\begin{array}{c}\text { USA } \\
(\mathrm{N}=178 \\
\text { Medien- und } \\
\text { Publicity- } \\
\text { Frames })\end{array}$ & $\begin{array}{c}\text { UK } \\
(\mathrm{N}=44 \\
\text { Medien- und } \\
\text { Publicity- } \\
\text { Frames })\end{array}$ & $\begin{array}{c}\text { GER } \\
(\mathrm{N}=96 \\
\text { Medien- und } \\
\text { Publicity- } \\
\text { Frames })\end{array}$ & $\begin{array}{c}\text { Gesamt: } \\
\text { Durchschnitts- } \\
\text { häufigkeit der } \\
\text { Frametypen } \\
\text { über alle Länder }\end{array}$ \\
\hline $\begin{array}{c}\text { Medien-Frames: } \\
\text { Vermittler }\end{array}$ & 25 & 50 & 45 & 40 \\
Strategie & 10 & 23 & 15 & 16 \\
$\quad$ Verantwortlichkeit & 3 & 2 & 12 & 6 \\
\hline Publicity-Frames: & 10 & 0 & 3 & 4 \\
$\quad$ Vermittler & 39 & 20 & 20 & 26 \\
$\quad$ Strategie & 13 & 5 & 6 & 100 \\
\hline Verantwortlichkeit & 100 & 100 & 101 & 8 \\
\hline Total & & & & \\
\hline
\end{tabular}

\subsection{Forschungsfrage: Frameverknüpfung zwischen Metaberichterstattung und übriger Wahlberichterstattung}

Metaberichterstattungsframes sind, wie gesehen, besonders eng mit den Themen Skandalisierung/Fehler, Persönlichkeit/Charakter, Wahlkampf/Kampagne sowie Umfragen/Bevölkerungsmeinung verzahnt. Im Folgenden geht es nun um einige Auffälligkeiten bei Frameverknüpfungen mit diesen vier Themen. Werden Skandalisierungen zum Wahlkampfthema, ist der dominierende Medienframe jener des potenten, resonanzenreichen strategischen Akteurs, von dem Handlungskonsequenzen für andere ausgehen (Medien-Strategieframes). Allerdings kommt es auch zu reflektierenden, aufklärerischen Analysen der Medienbeteiligung (MedienVerantwortlichkeitsframes). Bei der Darstellung der Publicity-Maßnahmen skandalisierter Akteure überwiegen die taktischen Kalküle (Publicity-Strategieframes). 
Tabelle 6: Detailverknüpfungen zwischen Metaberichterstattungsframes und übrigen Wahlkampfthemen

\begin{tabular}{|c|c|c|c|c|c|c|c|c|c|c|c|c|}
\hline & \multicolumn{3}{|c|}{$\begin{array}{c}\text { Skandalisierung/ } \\
\text { Fehler }\end{array}$} & \multicolumn{3}{|c|}{$\begin{array}{c}\text { Persönlichkeit/ } \\
\text { Charakter }\end{array}$} & \multicolumn{3}{|c|}{$\begin{array}{l}\text { Wahlkampf/ } \\
\text { Kampagne }\end{array}$} & \multicolumn{3}{|c|}{$\begin{array}{c}\text { Umfragen/ } \\
\text { Bev.-meinung }\end{array}$} \\
\hline & US & UK & $\mathrm{D}$ & US & UK & $\mathrm{D}$ & US & UK & $\mathrm{D}$ & US & UK & $\mathrm{D}$ \\
\hline $\begin{array}{l}\text { Häufigkeit }(N) \\
\text { des Themas in } \\
\text { der Bericht- } \\
\text { erstattung* }\end{array}$ & 10 & 14 & 22 & 54 & 20 & 22 & 161 & 159 & 102 & 98 & 34 & 46 \\
\hline \multicolumn{13}{|c|}{ Prozentuale Häufigkeit der Verknüpfung mit... } \\
\hline $\begin{array}{l}\text {...Medien- } \\
\text { Vermittlungs- } \\
\text { frame } \\
\end{array}$ & 10 & 21 & 4 & 4 & 5 & 4 & 6 & 4 & 20 & 30 & 24 & 39 \\
\hline $\begin{array}{l}\text {...Medien- } \\
\text { Strategieframe }\end{array}$ & 50 & 7 & 50 & 11 & 15 & -- & 4 & 4 & 2 & -- & -- & 2 \\
\hline $\begin{array}{l}\text {...Medien- } \\
\text { Verantwort- } \\
\text { lichkeitsframe }\end{array}$ & 20 & -- & 14 & -- & -- & 4 & 1 & 1 & 4 & 1 & -- & 4 \\
\hline $\begin{array}{l}\text {...Publicity- } \\
\text { Vermittlungs- } \\
\text { frame } \\
\end{array}$ & -- & -- & -- & 4 & -- & -- & 8 & -- & 3 & 1 & -- & -- \\
\hline $\begin{array}{l}\text { Publicity - } \\
\text { Strategieframe }\end{array}$ & 20 & -- & 18 & 40 & 15 & 14 & 24 & 4 & 12 & 3 & -- & -- \\
\hline $\begin{array}{l}\text {...Publicity- } \\
\text { Verantwort- } \\
\text { lichkeitsframe }\end{array}$ & -- & -- & 9 & -- & -- & 4 & 8 & 1 & 3 & 3 & -- & -- \\
\hline
\end{tabular}

* Angaben basieren auf 472 Themennennungen in den amerikanischen, 350 in den britischen und 269 in den deutschen TV-Wahlkampfnachrichten.

Stehen Persönlichkeit/Charakter im Mittelpunkt der Wahlkampfberichterstattung, werden häufig deren Image- und Werbebemühungen mit strategischen Publicityframes berichtet. Einen strategischen Medienframe gibt es in diesem Kontext vor allem dann, wenn die Rolle der Medien im taktischen Kalkül der Kandidaten thematisiert wird oder wenn sich Kandidaten über die Medienberichterstattung zu ihrer Person beklagten. Sehr häufig werden auch Aspekte von Wahlkampf/Kampagne mit Metaberichterstattung kombiniert. Hierbei werden die Medien- und Publicity-Bezüge allerdings in nahezu sämtlichen möglichen Rollen (bzw. Frames) gezeigt, sodass trotz enger Kopplung nur wenige charakterische Muster aufscheinen. Schließlich werden Umfragen besonders häufig mit Medien-Vermittlungsframes verknüpft, indem Nachrichtensender beispielsweise hervorheben, dass die präsentierten Meinungsumfragen „ihre“ Ergebnisse sind. Sie präsentieren sie mit vermittlungsbetonenden Einbindungen wie: „And here the latest from our NBC 
tracking poll; as our figures show the race is still down to the wire....". Insgesamt bilanzieren wir, dass trotz der etwas stärkeren Strategiebetonung der USFernsehnachrichten die Befunde wiederum für eine Hybridisierung der Berichterstattungsstile sprechen. Positiv für Deutschland ist bei den hier untersuchten Verknüpfungen zu vermerken, dass dort etwas häufiger als in den Vergleichsländern der Verantwortlichkeitsframe zu beobachten war (erwartungsgemäß öfters bei der öffentlich-rechtlichen ARD als bei der privaten Konkurrenz von RTL).

\section{$7 \quad$ Fazit und Folgerungen}

Der Mediatisierungsprozess verweist auf einen Struktur-, Kultur- und Diskurswandel im politischen Kommunikationssystem vieler Länder (Abschnitt 1). Die Grundannahme der vorliegenden Studie lautet, dass ein struktur- und kulturbedingt hoher Grad der Medienorientierung im politischen Kommunikationssystem zu intensiver Metaberichterstattung führt (Abschnitte 2, 3). Der strukturbedingte Mediatisierungsgrad wurde über verschiedene verfügbare Indikatoren erfasst ( $\mathrm{Ab}$ schnitt 4.1), ebenso der kulturbedingte (Abschnitt 4.2). Eine auf Basis dieser beiden unabhängigen Variablenbündel vorgenommene Rankingeinschätzung ergab einen hohen Mediatisierungsgrad des US-Kommunikationssystems zum Zeitpunkt der Präsidentschaftswahl 2000, einen mittleren Mediatisierungsgrad des deutschen Kommunikationssystems zum Zeitpunkt der Bundestagswahl 2002 und einen moderaten Mediatisierungsgrad während der britischen Unterhauswahl 2001. Aufgrund der unterschiedlichen Ausprägungen auf Seite der unabhängigen Variablen gingen diese Länder in die Untersuchung ein. Das gewählte SS-DO Design erwartete entsprechende unterschiedliche Ausprägungen auf Seite der abhängigen Variable (Abschnitt 5). Metaberichterstattung wurde definiert als Medienselbstthematisierung und Publicitythematisierung im Wahlkampf. Dabei wurde angenommen, dass die Rolle der Medien und die der Publicity mit spezifischen Frames und mit spezifischen Themenumfeldern kombiniert werden. Die theoretischen Grundlagen für diese Annahmen wurden aus der Nachrichtentheorie, der journalistischen Professions- und Rollentheorie, der Lerntheorie, der Theorie der Medien- und Aufmerksamkeitsökonomie, der Sozialverantwortlichkeits- und Demokratietheorie sowie der Konflikt- und Interaktionstheorie zwischen Journalismus und PR bzw. Medien und Politik entwickelt. Im Bestreben, Einsichten zur Validierung, Generalisierbarkeit und Kontextabhängigkeit des Metaberichterstattungskonzept zu gewinnen, wurden vier Hypothesen und eine Forschungsfrage entwickelt. 
Die erste Hypothese (das Niveau der Metaberichterstattung ist in den amerikanischen Fernsehnachrichten am höchsten und in den britischen am niedrigsten) wird bestätigt. Das Niveau der Metaberichterstattung scheint weitgehend eine Funktion der genannten unabhängigen Variablen zu sein. Allerdings kam es in Deutschland $\mathrm{zu}$ einer überraschend intensiven Metaberichterstattung, die fast an das amerikanische Niveau heranreichte. Dies ließ sich durch mehrere medien- und publicity-relevante Innovationen im Strukturinventar des politischen Kommunikationssystems erklären. Daraus ist zu folgern, dass Metaberichterstattung nicht nur eine evolutionäre diskursive Reaktion auf den strukturellen und kulturellen Mediatisierungsanstieg ist, sondern auch die Form kurzfristiger Impulsreaktionen auf Schlüsselereignisse annehmen kann. Offensichtlich ist Metaberichterstattung genauso ereignis- und aktualitätszentriert wie andere Genres des Nachrichtenjournalismus.

Die zweite Hypothese (das Themenumfeld der Metaberichterstattung ist in den amerikanischen Fernsehnachrichten stärker von einer Orientierung an Wahlkampf/Kampagnenführung, Umfragen/Bevölkerungsmeinung, Persönlichkeit/ Charakter und Skandalisierung/Fehler sowie einer Dethematisierung von Inhalte/Positionen und Ideologie/Weltsicht geprägt als in den deutschen und britischen Fernsehnachrichten) wird nicht bestätigt. Trotz vieler Unterschiede im institutionellen Arrangement der politischen Kommunikationssysteme (bedingt durch verschiedene Regierungs-, Parteien-, Wahl-, und Mediensysteme) scheinen sich die Handlungsorientierungen und Nachrichtenwerte der Politikjournalisten anzugleichen. Dies interpretieren wir ebenfalls als Hinweis darauf, dass Kommunikationsstrukturen und Kommunikationskulturen sich nicht gegenseitig voll determinieren und nicht eins zu eins auseinander ableitbar sind. Vielmehr scheint sich im Nachrichtenjournalismus westlicher Massendemokratien ein Set ähnlich gelagerter Orientierungen und Werte $\mathrm{zu}$ formieren. Die hier behauptete Angleichungstendenz (die durch Langzeitanalysen abgesichert werden müsste) ließe sich erklären durch die intensive gegenseitige Koorientierung unter internationalen Fernsehstationen, die transnationale Diffusion von Medienformaten, und die Übernahme von - medienzentrierten, politikdistanten - Berichterstattungsoptionen, wenn sie den professionellen und ökonomischen Eigeninteressen des Berufsstandes entsprechen.

Die dritte Hypothese (Metaberichterstattung wird vor allem verknüpft mit Kampagnen-, Umfrage-, Personalisierungs- und Skandalisierungs-Themen) wird für alle drei Länder gleichermaßen bestätigt. Als universelles Feature von Metaberichterstattung kann festgehalten werden, dass es im Wahlkampfkontext vor allem an Process- und Personality-Themen (die Politikwissenschaft spricht von Politics) gebunden ist. Weil Metaberichterstattung jedoch nicht in Policy-Beiträgen ver- 
wendet wird, scheint die Befürchtung einer Verdrängung von substanziellen Politikinhalten unbegründet.

Die vierte Hypothese (in der amerikanischen Metaberichterstattung überwiegen Strategieframes, in der deutschen und britischen Verantwortlichkeitsframes) wird hinsichtlich der Strategieframes nur schwach, hinsichtlich der Verantwortlichkeitsframes gar nicht bestätigt. Zwar überwiegt erwartungsgemäß die Strategieorientierung im hochmediatisierten amerikanischen Kommunikationssystem, aber beim überall geringen Vorkommen der Verantwortlichkeitsframes versagt die Erklärungskraft der zentralen unabhängigen Variablen. Trotz der Gemeinwohlverpflichtung des starken öffentlich-rechtlichen Rundfunks in Großbritannien und Deutschland waren Verantwortlichkeitsframes dort genauso selten wie in den USA. Am häufigsten wurden überall Vermittlungsframes verwendet. Wir interpretieren diese Ergebnisse als weiteren Hinweis auf Elemente einer westlichen Nachrichtenkultur: Erstens gleicht sich die Strategieorientierung überall aus den oben genannten Gründen an. Zweitens hat der Journalismus überall ein professionelles Eigeninteresse daran, die Rolle der Medien eher in der neutralen Vermittler- als in der aktiven Strategierolle darzustellen (die Vermittlerrolle ist weniger legitimationsbedürftig). Schließlich scheint die professionsethische Veranlagung für Verantwortlichkeitsframes überall vorhanden, ihre Anwendung jedoch wiederum stark ereignis- und aktualitätsabhängig zu sein. Weil es in den hier untersuchten Wahlkämpfen an konkreten Anlässen mangelte, die einen analytischen, kritischen, aufklärerischen Umgang mit Grenzverletzungen der Medien und Publicity erforderlich machten, lag deren Niveau entsprechend niedrig.

Im Sinne der Konzeptvalidierung können damit einige Konstanten der Metaberichterstattung festgestellt werden: Erstens scheint das Niveau der Metaberichterstattung eine weitgehend lineare Funktion der unabhängigen Variablen (struktureller und kultureller Mediatisierungsgrad) zu sein. Zweitens kann es durch kurzfristigen Strukturwandel (medien- und publicity-bezogene Innovationen) zu Eruptionen der Metaberichterstattung kommen. Drittens ist Metaberichterstattung an die von der normativen Demokratietheorie wenig geschätzten Themenfelder Process und Personality gebunden. Viertens scheint der Journalismus bei Medienselbstthematisierungen ein professionelles Eigeninteresse daran zu haben, die Rolle der Medien eher in der neutralen Vermittler- als in der aktiven Strategierolle darzustellen. Fünftens scheint die Bereitschaft zur Verwendung von Verantwortlichkeitsframes in allen Nachrichtenkulturen verankert; ihre Aktivierung ist jedoch von konkreten Grenzverletzungen abhängig. Gerade durch die Möglichkeit der Anwendung von Verantwortlichkeitsframes erscheint uns Metaberichterstattung aber als ein spannender und gewinnbringender Beitrag zur Wahlberichterstattung. 
Sechstens scheint Metaberichterstattung ein typisches Ergebnis von hybriden Wahlkampfstilen zu sein, bei denen nationale, länderspezifische Kampagnen- und Berichterstattungstraditionen um ausgewählte Komponenten eines medien- und publicity-zentrierten „hyper-reflexiven“ Stils (Moog/Slyter-Beltro 2000: 38) ergänzt werden. Wie valide diese Schlussfolgerungen sind, muss die weitere Forschung im ausgedehnten Zeit- und Ländervergleich zeigen.

\section{Literatur}

Althaus, Marco/Cecere, Vito (Hrsg.) (2003): Kampagne 2. Neue Strategien für Wahlkampf, PR, Lobbying. Münster: Lit.

Altheide, David L./Snow, Robert P. (1991): Media worlds in the post-journalism era. New York, NY: DeGruyter.

Axford, Barrie/Huggins, Richard (Hrsg.) (2001): New media and politics. London: Sage.

Bennett, W. Lance/Entman, Robert M. (Hrsg.) (2001): Mediated politics. Communication and the future of democracy. New York: Cambridge University Press.

Bentele, Günter/Brosius, Hans-Bernd/Jarren, Otfried (Hrsg.) (2003): Öffentliche Kommunikation. Handbuch Kommunikations- und Medienwissenschaft. Wiesbaden: Westdeutscher Verlag.

Berg, Stefan (2002): Die Politiker und das Mehr. Die Aufregung um die Bonusmeilen hat alle anderen Wahlkampfthemen in den Hintergrund gedrängt. In: Der Spiegel 32 (5. August 2002): 22-33.

Bergmann, Knut (2002): Der Bundestagswahlkampf 1998. Vorgeschichte, Strategien, Ergebnis, Wiesbaden: Westdeutscher Verlag.

Blumler, Jay G. (1990): Elections, the media and the modern publicity process. In: Fergurson (1990): 101113.

Blumler, Jay G. (1997): Origins of the crisis of communication for citizenship. In: Political Communication 14: 395-404.

Blumler, Jay G./Gurevitch, Michael (1995): The crisis of public communication. London: Routledge.

Blumler, Jay G./Gurevitch, Michael (2001): “Americanization" reconsidered. U.K.-U.S. campaign communication across time. In: Bennett/Entman (2001): 380-403.

Blumler, Jay G./Kavanagh, Dennis (1999): The third age of political communication. Influences and features. In: Political Communication 16 (3): 209-230.

Bohrmann, Hans/Jarren, Otfried/Melischek, Gabriele/Seethaler, Josef (Hrsg.) (2000): Wahlen und Politikvermittlung durch Massenmedien. Wiesbaden: Westdeutscher Verlag.

Butler, David/Kavanagh, Dennis (2002): The British general election of 2001. Houndmills: Palgrave.

D'Angelo, Paul (2002): News framing as a multi-paradigmatic research program. A response to Entman. In: Journal of Communication 52 (4): 870-888.

D'Angelo, Paul/Esser, Frank (2003): Metacoverage of the press and publicity in Campaign 2000 Network News. In: Kaid et al. (2003): 89-103.

Dahlgren, Peter/Sparks, Colin (Hrsg.) (1991): Communication and citizenship. London: Routledge.

Donges, Patrick (2005): Medialisierung der Politik? Vorschlag einer Differenzierung. In: Rössler/Krotz (2005): 321-339.

Dulio, David A. (2003): Inside the war room. Political consultants in modern campaigns. In Watson/Campbell (2003): 17-29.

Electoral Commission (2002): Election 2001: Campaign Spending. London: Electoral Commission. http://www.electoralcommission.org.uk/elections/2001report.cfm [Abruf: 13. Juni 2007]. 
Entman, Robert M. (1993): Framing. Towards clarification of a fractured paradigm. In: Journal of Communication 43 (4): 51-58.

Esser, Frank/D'Angelo, Paul (2003): Framing the press and the publicity process. A content analysis of metacoverage in campaign 2000 Network News. In: American Behavioral Scientist 46 (5): 617-641.

Esser, Frank/Pfetsch, Barbara (Hrsg.) (2003): Politische Kommunikation im internationalen Vergleich. Grundlagen, Anwendungen, Perspektiven. Wiesbaden: Westdeutscher Verlag.

Esser, Frank/Reinemann, Carsten/Fan, David P. (2001): Spin doctors in the United States, Great Britain and Germany. Metacommunication about media manipulation. In: Harvard International Journal of Press/Politics 6 (1): 16-45.

Farnsworth, Stephen J./Lichter, Robert S. (2003): The nightly news nightmare. Lanham, MD: Rowman and Littlefield.

Fergurson, Marjorie (Hrsg.) (1990): Public Communication. The new imperatives. London: Sage.

Filzmaier, Peter/Plasser, Fritz (2001): Wahlkampf um das Weisse Haus. Presidential Elections in den USA. Opladen: Leske + Budrich.

Fisher, Justin (2001): Campaign finance. In: Parliamentary Affairs 54: 689-700.

Gamson, William A. (1989): News as framing. In: American Behavioral Scientist 33 (2): 157-161.

Geddes, Anthony/Tonge, James (Hrsg.) (2002): Labour's second landslide. The British general election of 2001. Manchester: Manchester University Press.

Genz, Andreas/Schönbach, Klaus/Semetko, Holli A. (2001): Amerikanisierung? Politik in den Fernsehnachrichten während der Bundestagswahlkämpfe 1990-1998. In: Klingemann/Kaase (2001): 401414.

Gitlin, Todd (1991): Blips, bytes and savvy talk. Television's impact on American politics. In: Dahlgren/Sparks (1991): 119-136.

Goldstein, Ken/Freedman, Paul (2002): Lessons learned. Campaign advertising in the 2000 elections. In: Political Communication 19 (1): 5-28.

Graber, Doris (2007): Mass media and American politics. 7. Auflage. Washington, DC: CQ Press.

Graber, Doris/McQuail, Denis/Norris, Pippa (Hrsg.) (1998): The politics of news, the news of politics. Washington: CQ Press.

Gunther, Richard/Mughan, Anthony (Hrsg.) (2000): Democracy and the media. A comparative perspective. New York: Cambridge University Press.

Gurevitch, Michael/Blumler, Jay G. (2003): Der Stand der vergleichenden politischen Kommunikationsforschung. Ein eigenständiges Feld formiert sich. In Esser/Pfetsch (2003): 371-392.

Hallin, Dan (1992): Sound-bite news. Television coverage of elections, 1968-1988. In: Journal of Communication 42: 5-24.

Harrison, Martin (2002): Politics on the air. In: Butler/Kavanagh (2002): 132-155.

Hartmann, A./Hones, B./Schultz, H. (2003): Leere Kassen. In: Focus Money 11. Dezember: 71-75.

Holtz-Bacha, C. (2005): To the advantage of the big parties. TV advertising during the 2002 German national election campaign. In: Journal of Political Marketing 4 (4): 75-84.

Jahn, Detlef (2006): Einführung in die vergleichende Politikwissenschaft. Opladen: Leske und Budrich.

Jarren, Otfried/Donges, Patrick (2006): Politische Kommunikation in der Mediengesellschaft. Eine Einführung. 2. überarbeitete Auflage. Wiesbaden: VS Verlag für Sozialwissenschaften.

Johnson, Thomas J./Boudreau, Timothy/Glowaki, Chris (1996): Turning the spotlight inward. How five leading news organizations covered the media in the 1992 presidential election. In: Journalism und Mass Communication Quarterly 73 (3): 657-671.

Kaid, Lynda Lee/Tedesco, John C./Bystrom, Dianne/McKinney, Mitchell S. (Hrsg.) (2003): The millennium election. Communication in the 2000 campaigns. Lanham, MD: Rowman and Littlefield.

Kepplinger, Hans Mathias (1990): Realität, Realitätsdarstellung und Medienwirkung. In: Wilke (1990): 39-55. 
Kepplinger, Hans Mathias (1998): Die Demontage der Politik in der Informationsgesellschaft. Freiburg: Alber.

Kepplinger, Hans Mathias (2001): Der Ereignisbegriff in der Publizistikwissenschaft. In: Publizistik 46 (2): $117-139$.

Kerbel, Matthew Robert (1997): The media. Viewing the campaign through a strategic haze. In: Nelson (1997): 81-105.

Kerbel, Matthew Robert (1998): Edited for television. CNN, ABC, and American presidential politics. 2. Auflage. Boulder, CO: Westview Press.

Kerbel, Matthew Robert (1999): Remote and controlled. Media politics in a cynical age. 2. überarbeitete Auflage. Boulder: Westview Press.

Kerbel, Matthew Robert/Apee, Sumaiya/Ross, Marc Howard (2000): PBS ain't so different. Public broadcasting, election frames, and democratic empowerment. In: Harvard International Journal of Press/ Politics 5 (4): 8-32.

Klingemann, Hans-Dieter/Kaase, Max (Hrsg.) (2001): Analysen aus Anlass der Bundestagswahl 1998. Wiesbaden: Westdeutscher Verlag.

Kuhn, Raymond/Neveu, Eric (Hrsg.) (2002): Political journalism. New challenges, new practices. London: Routledge.

Magleby, David B. (Hrsg.) (2002): Financing the 2000 election. Washington, DC: Brookings Institute.

Mazzoleni, Gianpietro/Schulz, Winfried (1999): Mediatization of politics. A challenge for democracy? In: Political Communication 16 (3): 247-261.

McNair, Brian (2000): Journalism and democracy. An evaluation of the political public sphere. London: Routledge.

McQuail, Denis (2005): McQuail's mass communication theory. 5. Auflage. London: Sage.

Moog, Sandra/Sluyter-Beltro, Jeffrey (2001): The transformation of political communication? In: Axford/ Huggins (2001): 30-63.

Müller, Dieter K. (2002): Wahlwerbung im Fernsehen. ARD und ZDF als Werbeträger nach 20 Uhr. In: Media Perspektiven 12: 623-628.

Müller, Marion (2002): Parteienwerbung im Bundestagswahlkampf 2002. In: Media Perspektiven 12: 629638.

Nelson, Candice J. (2002): Spending in the 2000 elections. In: Magleby (2002): 22-48.

Nelson, Michael (Hrsg.) (1997): The election of 1996. Washington, DC: CQ Press.

Neveu, Eric (2002): Four generations of political journalism. In: Kuhn/Neveu (2002): 22-43.

Pan, Zhongdang/Kosicki, Gerald M. (1993): Framing analysis. An approach to news discourse. In: Political Communication 10 (1): 55-76.

Patterson, Thomas E. (1993): Out of order. New York: Knopf.

Pfetsch, Barbara (1998): Government news management. In: Graber et al. (1998): 70-93.

Pfetsch, Barbara (2003): Politische Kommunikationskultur. Ein theoretisches Konzept zur vergleichenden Analyse politischer Kommunikationssysteme. In: Esser/Pfetsch (2003): 393-418.

Pfetsch, Barbara (2003): Politische Kommunikationskultur. Politische Sprecher und Journalisten in der Bundesrepublik und den USA im Vergleich. Wiesbaden: Westdeutscher Verlag.

Pfetsch, Barbara/Esser, Frank (2003): Politische Kommunikation im internationalen Vergleich. Neuorientierung in einer veränderten Welt. In: Esser/Pfetsch (2003): 9-31.

Plasser Fritz/Plasser, Gunda (2003): Globalisierung der Wahlkämpfe. Praktiken der Campaign Professionals im weltweiten Vergleich. Wien: WUV-Universitätsverlag.

Przeworski, Adam/Teune, Henry (1970): The logic of comparative social inquiry. Malabar, FL: Krieger.

Reese, Stephen D. (2001): Prologue - framing public life. A bridging model for media research. In: Reese et al. (2001): 7-31. 
Reese, Stephen/Gandy, Oscar H./Grant, A. E. (Hrsg.) (2001): Framing public life. Perspectives on media and our understanding of the social world. Mahwah, NJ: Lawrence Erlbaum.

Röseler, Oliver (2003): Wahlkampforganisation der CDU/CSU: Union wie noch nie. In: Althaus/Cecere (2003): 199-211.

Rössler, Patrick/Krotz, Friedrich (Hrsg.) (2005): Mythen der Mediengesellschaft. The media society and its myths. Konstanz: UVK.

Sarcinelli, Ulrich (2000). Politikvermittlung und Wahlen. Sonderfall oder Normalität des politischen Prozesses. In Bohrmann et al. (2000): 19-30.

Scheufele, Bertram (2003): Frames, Framing, Framing-Effekte. Theoretische Grundlegung, methodische Umsetzung sowie empirische Befunde zur Nachrichtenproduktion. Wiesbaden: Westdeutscher Verlag.

Scheufele, Dietram A. (1999): Framing as a theory of media effects. In: Journal of Communication 49 (1): 103-122.

Scheufele, Dietram/Tewsbury, David (2007): Framing, Agenda Setting and priming. In: Journal of Communication 57 (1): 9-20.

Schulz, Winfried (2003): Politische Kommunikation. In: Bentele et al. (2003): 458-480.

Semetko, Holli A. (1996): Political balance on television. Campaigns in the United States, Britain, and Germany. In: Harvard International Journal of Press/Politics 1 (1): 51-71.

Semetko, Holli A. (2000): Great Britain. The end of „News at Ten“ and the changing news environment. In: Gunther/Mughan (2000): 343-374.

Semetko, Holli. A./Blumler, Jay G./Gurevitch, Michael/Weaver, David H. (1991): The formation of campaign agendas. A comparative analysis of party and media roles in recent American and British elections. Hillsdale, NJ: Lawrence Erlbaum.

Shoemaker, Pamela/Reese, Stephen D. (1996): Mediating the message. Theories of influences on mass media content. 2. Auflage. White Plains, NY: Longman.

Tankard, James W. (2001): The empirical approach to the study of media framing. In: Reese et al. (2001): 95-106.

Watson, Robert P./Campbell, Colton C. (Hrsg.) (2003): Campaigns and elections. Issues, concepts, cases. Boulder, CO: Lynne Rienner.

Weaver, David (1998): The global journalist. News people around the world. Creskill, NJ: Hampton Press.

Wilke, Jürgen (Hrsg.) (1990): Fortschritte der Publizistikwissenschaft. Freiburg: Alber.

Wilke, Jürgen/Reinemann, Carsten (2001): Do the candidates matter? Long-term trends of campaign coverage. A study of the German press since 1949. In: European Journal of Communication 16 (3): 291314 .

Wring, Dominic (2002): The Tony press. Media coverage of the election campaign. In: Geddes/Tonge (2002): 84-101.

Zaller, John (1999): A theory of media politics. How the interests of politicians, journalists and the citizens shape the news. Book manuscript under contract to University of Chicago Press (draft version available at http://www.polisci.ucla.edu/faculty/zaller/).

Zubayr, Camille/Gerhard, Heinz (2002): Berichterstattung zur Bundestagswahl 2002 aus Sicht der Zuschauer. In: Media Perspektiven 12: 586-599. 